\title{
UV(IUE) spectra of hot post-AGB candidates ${ }^{\star \star \star}$
}

\author{
G. Gauba and M. Parthasarathy
}

\begin{abstract}
Indian Institute of Astrophysics, Koramangala, Bangalore 560034, India
Received 25 April 2003 / Accepted 3 June 2003

Abstract. Analysis of the low resolution UV(IUE) spectra (1150 to $3200 \AA$ ) of 15 hot post-AGB candidates is presented. The UV(IUE) spectra of 10 stars suggest partial obscuration of the hot stars due to circumstellar dust. The reddened continua of these 10 stars were used to model and estimate the circumstellar extinction. The circumstellar extinction law was found to be linear in $\lambda^{-1}$ in the case of IRAS 13266-5551 (CPD-55 5588), IRAS 14331-6435 (Hen3-1013), IRAS 16206-5956 (SAO 243756), IRAS 17074-1845 (Hen3-1347), IRAS 17311-4924 (Hen3-1428), IRAS 18023-3409 (LSS 4634), IRAS 18062+2410 (SAO 85766), IRAS 18371-3159 (LSE 63), IRAS 22023+5249 (LSIII +5224) and IRAS 22495+5134 (LSIII +5142). There seems to be no significant circumstellar extinction in the case of IRAS 17203-1534, IRAS 17460-3114 (SAO 209306) and IRAS 183791707 (LSS 5112). The UV(IUE) spectrum of IRAS 12584-4837 (Hen3-847) shows several emission lines including that of HeII. It may be a massive young OB-supergiant or a low mass star in the post-AGB phase of evolution. IRAS 16206-5956 (SAO 243756) and IRAS $18062+2410$ (SAO 85766) show variability in the UV which in addition to stellar pulsations may be attributed to a dusty torus in motion around the hot central stars. The UV spectrum of the bipolar PPN, IRAS 17423-1755 (Hen3-1475) indicates that the central B-type star is obscured by a dusty disk. The stars were placed on the $\log g-\log T_{\text {eff }}$ diagram showing the post-AGB evolutionary tracks of Schönberner. Terminal wind velocities of the stars were estimated from the CIV and NV stellar wind features. The presence of stellar wind in some of these stars indicates ongoing post-AGB massloss.
\end{abstract}

Key words. stars: AGB and post-AGB - stars: early-type - stars: evolution - stars: circumstellar matter - ultraviolet: stars

\section{Introduction}

Low and intermediate mass stars $\left(M \simeq 0.8-8 M_{\odot}\right)$ pass through the post-asymptotic giant branch (post-AGB) phase of evolution on their way to becoming planetary nebulae $(\mathrm{PNe})$. From an analysis of the Infrared Astronomical Satellite Point Source Catalog (IRAS PSC), cooler post-AGB stars having G, F, A supergiant-like character were first identified (Parthasarathy \& Pottasch 1986; Lamers et al. 1986; Pottasch \& Parthasarathy 1988a; Hrivnak et al. 1989). These stars were found to have circumstellar dust shells with far-IR colors and flux distributions similar to the dust shells of PNe. Later, from an analysis of IRAS data, Parthasarathy \& Pottasch (1989) found a few hot (OB spectral types) post-AGB candidates. Their supergiant-like character, the presence of cold detached dust shells, far-IR colors similar to PNe and high galactic latitudes suggested that they may be in a post-AGB phase of evolution. Thus, there seems to be an evolutionary sequence ranging from the cooler G, F, A supergiant-like stars to hotter O-B types, evolving from the tip of the AGB towards young PN stage (Parthasarathy 1993c).

Send offprint requests to: $\mathrm{G}$. Gauba,

e-mail: gauba@iiap.ernet.in

* Based on observations obtained with the International Ultraviolet Explorer (IUE), retrieved from the Multimission Archive at STScI.

$\star \star$ Complete Figs. 3 and 4 and Appendix A are only available in electronic form at http://www . edpsciences .org
Pottasch et al. (1988b) and van der Veen \& Habing (1988) identified a region of the IRAS color-color diagram $(F(12 \mu) / F(25 \mu)<0.35$ and $F(25 \mu) / F(60 \mu)>0.3)$ which was mainly populated by stars in transition from the AGB to the PN phase. Based on their far-IR colors and low resolution optical spectra, several hot post-AGB candidates were identified (Parthasarathy \& Pottasch 1989; Parthasarathy 1993a, 1993c; Parthasarathy et al. 2000a). The optical spectra of these objects show strong Balmer emission lines and in some cases low excitation nebular emission lines such as [NII] and [SII] superposed on the OB stellar continuum. The absence of [OIII] $5007 \AA$ Aine and the presence of low excitation nebular emission lines indicate that photoionisation has just started. It is important to study these stars in the UV to obtain better estimates of their temperatures and to look for signatures of circumstellar reddening, mass-loss and stellar winds. The UV(IUE) spectra of some hot post-AGB stars (e.g. Hen3-1357, Parthasarathy et al. 1993b, 1995; Feibelman 1995) have revealed violet shifted stellar wind P-Cygni profiles of CIV, SiIV and NV, indicating hot and fast stellar wind, post-AGB mass-loss and rapid evolution. In this paper we have analysed the UV(IUE) spectra of 15 hot post-AGB candidates.

\section{Target selection and observations}

The hot post-AGB candidates in this paper (Table 1) were identified on the basis of their IRAS colors $(F(12 \mu) / F(25 \mu)<0.35$ and $F(25 \mu) / F(60 \mu)>0.3)$, high galactic latitudes and 
Table 1. Hot post-AGB candidates.

\begin{tabular}{|c|c|c|c|c|c|c|c|c|}
\hline \hline Star No. & IRAS & Name & $l$ & $b$ & \multicolumn{4}{|c|}{ IRAS Fluxes (Jy.) } \\
\cline { 6 - 9 } & & & & & $12 \mu$ & $25 \mu$ & $60 \mu$ & $100 \mu$ \\
\hline 1. & $12584-4837$ & Hen3-847 & 304.60 & +13.95 & 36.07 & 48.75 & 13.04 & 3.31 \\
2. & $13266-5551$ & CPD-55 5588 & 308.30 & +6.36 & 0.76 & 35.90 & 35.43 & 11.66 \\
3. & $14331-6435$ & Hen3-1013 & 313.89 & -4.20 & 4.04 & 108.70 & 70.71 & 20.61 \\
4. & $16206-5956$ & SAO 243756 & 326.77 & -7.49 & $0.36 \mathrm{~L}$ & 11.04 & 12.30 & 4.83 \\
5. & $17074-1845$ & Hen3-1347 & 4.10 & +12.26 & 0.50 & 12.20 & 5.66 & $3.47:$ \\
6. & $17203-1534$ & & 8.55 & +11.49 & 0.32 & 10.70 & 6.88 & 3.37 \\
7. & $17311-4924$ & Hen3-1428 & 341.41 & -9.04 & 18.34 & 150.70 & 58.74 & 17.78 \\
8. & $17423-1755$ & Hen3-1475 & 9.36 & +5.78 & 7.05 & 28.31 & 63.68 & 33.43 \\
9. & $17460-3114$ & SAO 209306 & 358.42 & -1.88 & 6.26 & 20.82 & 12.20 & $220.40 \mathrm{~L}$ \\
10. & $18023-3409$ & LSS 4634 & 357.61 & -6.31 & $0.26 \mathrm{~L}$ & 2.94 & 1.82 & $25.64 \mathrm{~L}$ \\
11. & $18062+2410$ & SAO 85766 & 50.67 & +19.79 & 3.98 & 19.62 & 2.90 & $1.00 \mathrm{~L}$ \\
12. & $18371-3159$ & LSE 63 & 2.92 & -11.82 & $0.25 \mathrm{~L}$ & 6.31 & 5.16 & 1.95 \\
13. & $18379-1707$ & LSS 5112 & 16.50 & -5.42 & 1.67 & 23.76 & 7.12 & $3.66 \mathrm{~L}$ \\
14. & $22023+5249$ & LSIII +5224 & 99.30 & -1.96 & 1.02 & 24.69 & 14.52 & $3.93 \mathrm{~L}$ \\
15. & $22495+5134$ & LSIII +5142 & 104.84 & -6.77 & 0.54 & 12.37 & 7.18 & 3.12 \\
\hline
\end{tabular}

A colon: indicates moderate quality IRAS flux, "L" is for an upper limit.

OB-giant or supergiant spectra in the optical (Parthasarathy et al. 2000a) with Balmer lines in emission. Young massive OB-supergiants are not expected at high galactic latitudes and also they are not expected to have detached cold circumstellar dust shells. High galactic latitude OB supergiants with detached dust shells and far-IR colors similar to PNe were found to be in the post-AGB phase of evolution (Parthasarathy 1993c; Parthasarathy et al. 2000a).

Low resolution $(\sim 6-7 \AA)$, large aperture, UV(IUE) spectra of the hot post-AGB candidates from $1150 \AA$ to $3200 \AA$ were extracted from the Multimission Archive at STScI (Table 2). The spectra obtained by centering the stars in the $10^{\prime \prime} \times$ $23^{\prime \prime}$ aperture were processed using the IUE NEWSIPS (new spectral image processing system) pipeline which applies the signal weighted extraction technique (SWET) as well as the latest flux calibration and close-out camera sensitivity corrections. An increased signal-to-noise $(S / N)$ ratio of $10 \%-50 \%$ has been demonstrated for low dispersion IUE spectra reprocessed with the NEWSIPS software (Nichols \& Linsky 1996). Well exposed IUE NEWSIPS spectra have $S / N$ of $\sim 50$ while weak, high-background, under-exposed spectra have $S / N$ of 20 (Nichols et al. 1994; Nichols \& Linsky 1996). From our sample, IRAS 14331-6435 (Hen3-1013), IRAS 17074-1845 (Hen3-1347), IRAS 17203-1534, IRAS 18023-3409 (LSS 4634), IRAS 18379-1707 (LSS 5112) and IRAS 22023+5249 (LSIII +5224) have $S / N \sim 20$. IRAS 12584-4837 (Hen3847), IRAS 17460-3114 (SAO 209306) and IRAS 18371-3159 (LSE 63) have well exposed spectra with $S / N \sim 50$. The spectra of the remaining hot post-AGB candidates are of intermediate quality with $S / N \sim 30$. The LWP spectra 18412 and 27936 of IRAS 12584-4837 and IRAS 18371-3159 respectively, were saturated and have not been used in the analysis. Line-by-line images were inspected for spurious features.

\section{Analysis}

Tables 3, 4a and $4 \mathrm{~b}$ list the $V$ magnitudes and $B-V$ values of the 15 hot post-AGB candidates from literature. The optical spectral types are mainly from Parthasarathy et al. (2000a). For the optical spectral types of the stars, the corresponding intrinsic $B-V$ values, $(B-V)_{0}$, were taken from Schmidt-Kaler (1982). Using the observed and intrinsic $B-V$ values we derived the total (interstellar plus circumstellar) extinction, $E(B-V)_{\text {total }}$ $\left(=(B-V)_{\text {obs }}-(B-V)_{0}\right)$ towards these stars. These values were compared with interstellar extinction $\left(E(B-V)_{\text {I.S. }}\right)$ at the galactic latitudes and longitudes of these stars, estimated using the Diffuse Infrared Background Experiment (DIRBE)/IRAS dust maps (Schlegel et al. 1998). The DIRBE/IRAS dust maps do not give reliable estimates of the interstellar extinction for $|b|<5^{\circ}$. The accuracy of the DIRBE/IRAS extinction estimates are $16 \%$. Comparing $E(B-V)_{\text {total }}$ and $E(B-V)_{\text {I.S. }}$ we found considerable circumstellar extinction in most cases (Tables $4 \mathrm{a}$ and $\mathrm{b}$ ).

The $2200 \AA$ feature in the UV gives an estimate of the interstellar extinction. The merged LWP and SWP spectra of the hot post-AGB candidates were dereddened (Tables 3, 4a and b) using the $2200 \AA$ feature in the UV. Using the UNRED routine in the IUE data analysis software package, we adopted the average extinction law by Seaton (1979) and tried different values of $E(B-V)$ till the $2200 \AA$ bump in the UV disappeared and smooth continua from $1150 \AA$ to $3200 \AA$ were obtained for all the stars. In the absence of an LWP spectrum of IRAS 17460 3114 (SAO 209306), the SWP spectrum of the star was dereddened using $E(B-V)_{\text {total }}\left((B-V)_{\text {obs }}-(B-V)_{0}\right)=0.54$. In the rest of this paper, "dereddened spectra" would always refer to the observed IUE spectra corrected for interstellar extinction as determined from the $2200 \AA$ feature in the UV. 
Table 2. Log of observations.

\begin{tabular}{|c|c|c|c|c|c|}
\hline IRAS & Camera & Image & Aperture & Date obs & Exposure time(s) \\
\hline $12584-4837$ & SWP & 39271 & LARGE & 21 July 1990 & 2999.781 \\
\hline \multirow[t]{2}{*}{$=$ Hen $3-847$} & LWP & 18412 & LARGE & 21 July 1990 & 1799.659 \\
\hline & LWP & 19726 & LARGE & 10 Feb. 1991 & 299.704 \\
\hline \multirow{2}{*}{$\begin{aligned} & 13266-5551 \\
= & \mathrm{CPD}-555588\end{aligned}$} & SWP & 39270 & LARGE & 20 July 1990 & 1799.652 \\
\hline & LWP & 18411 & LARGE & 20 July 1990 & 1199.595 \\
\hline \multirow{2}{*}{$\begin{array}{c}14331-6435 \\
=\text { Hen3-1013 }\end{array}$} & SWP & 33601 & LARGE & 22 May 1988 & 2399.717 \\
\hline & LWP & 13295 & LARGE & 22 May 1988 & 1199.595 \\
\hline \multirow{6}{*}{$\begin{aligned} & 16206-5956 \\
= & \mathrm{SAO} 243756\end{aligned}$} & SWP & 33953 & LARGE & 21 July 1988 & 2399.717 \\
\hline & SWP & 50195 & LARGE & 12 March 1994 & 2399.716 \\
\hline & SWP & 50640 & LARGE & 28 April 1994 & 3599.844 \\
\hline & LWP & 13714 & LARGE & 21 July 1988 & 1199.595 \\
\hline & LWP & 26175 & LARGE & 19 August 1993 & 1019.781 \\
\hline & LWP & 27667 & LARGE & 12 March 1994 & 599.531 \\
\hline \multirow{2}{*}{$\begin{array}{c}17074-1845 \\
=\text { Hen3-1347 }\end{array}$} & SWP & 39276 & LARGE & 21 July 1990 & 1799.652 \\
\hline & LWP & 18418 & LARGE & 21 July 1990 & 599.531 \\
\hline \multirow[t]{2}{*}{ 17203-1534 } & SWP & 50657 & LARGE & 30 April 1994 & 2399.716 \\
\hline & LWP & 28020 & LARGE & 30 April 1994 & 1199.595 \\
\hline \multirow{4}{*}{ 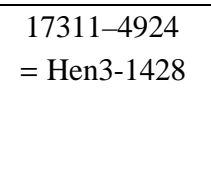 } & SWP & 33600 & LARGE & 21 May 1988 & 2099.480 \\
\hline & SWP & 48127 & LARGE & 17 July 1993 & 3599.844 \\
\hline & LWP & 13294 & LARGE & 22 May 1988 & 959.570 \\
\hline & LWP & 25934 & LARGE & 17 July 1993 & 899.768 \\
\hline $\begin{array}{c}17423-1755 \\
=\text { Hen3-1475 }\end{array}$ & SWP & 35860 & LARGE & 26 March 1989 & 2099.480 \\
\hline $\begin{aligned} & 17460-3114 \\
= & \text { SAO } 209306\end{aligned}$ & SWP & 54660 & LARGE & 12 May 1995 & 89.572 \\
\hline \multirow{2}{*}{$\begin{array}{l}18023-3409 \\
=\text { LSS } 4634\end{array}$} & SWP & 55458 & LARGE & 9 August 1995 & 4199.499 \\
\hline & LWP & 31272 & LARGE & 9 August 1995 & 1559.634 \\
\hline \multirow{4}{*}{$\begin{aligned} & 18062+2410 \\
= & \mathrm{SAO} 85766\end{aligned}$} & SWP & 44446 & LARGE & 21 April 1992 & 1200 \\
\hline & SWP & 55916 & LARGE & 12 Sep. 1995 & 1800 \\
\hline & LWP & 22865 & LARGE & 21 April 1992 & 300 \\
\hline & LWP & 31454 & LARGE & 12 Sep. 1995 & 600 \\
\hline \multirow{3}{*}{$\begin{array}{c}18371-3159 \\
=\text { LSE } 63\end{array}$} & SWP & 50588 & LARGE & 19 April 1994 & 4799.563 \\
\hline & LWP & 27936 & LARGE & 19 April 1994 & 2399.723 \\
\hline & LWP & 27972 & LARGE & 23 April 1994 & 899.768 \\
\hline \multirow{2}{*}{$\begin{array}{l}18379-1707 \\
=\text { LSS } 5112\end{array}$} & SWP & 47531 & LARGE & 23 April 1993 & 3899.672 \\
\hline & LWP & 25398 & LARGE & 23 April 1993 & 1199.595 \\
\hline \multirow{5}{*}{$\begin{array}{c}22023+5249 \\
=\text { LSIII }+5224\end{array}$} & SWP & 48454 & LARGE & 24 August 1993 & 2399.716 \\
\hline & SWP & 48593 & LARGE & 9 Sep. 1993 & 4799.563 \\
\hline & SWP & 55915 & LARGE & 12 Sep. 1995 & 7199.819 \\
\hline & LWP & 26209 & LARGE & 24 August 1993 & 1199.595 \\
\hline & LWP & 31453 & LARGE & 12 Sep. 1995 & 2399.723 \\
\hline \multirow{2}{*}{$\begin{aligned} & 22495+5134 \\
= & \text { LSIII +5142 }\end{aligned}$} & SWP & 48453 & LARGE & 24 August 1993 & 2399.716 \\
\hline & LWP & 26322 & LARGE & 9 Sep. 1993 & 2099.487 \\
\hline
\end{tabular}

For stars with multiple LWP and SWP spectra, we overplotted the observed LWP (and SWP) spectra of each star to check for variability. The LWP and SWP spectra of IRAS 162065956 (SAO 243756) and IRAS 18062+2410 (SAO 85766) were found to show variation (Fig. 1). Coadded LWP and SWP spectra of IRAS 17311-4924 and IRAS 22023+5249 were used in the analysis.
The dereddened merged spectra of the hot post-AGB candidates were compared with the dereddened spectra of standard stars from the atlas by Heck et al. (1984) The spectral types of the standard stars chosen for comparison were as close as possible to the optical spectral types of the hot postAGB candidates. Tables 3, $4 \mathrm{a}$ and $4 \mathrm{~b}$ list the standard stars used for comparison, their spectral types and $E(B-V)$ values. 

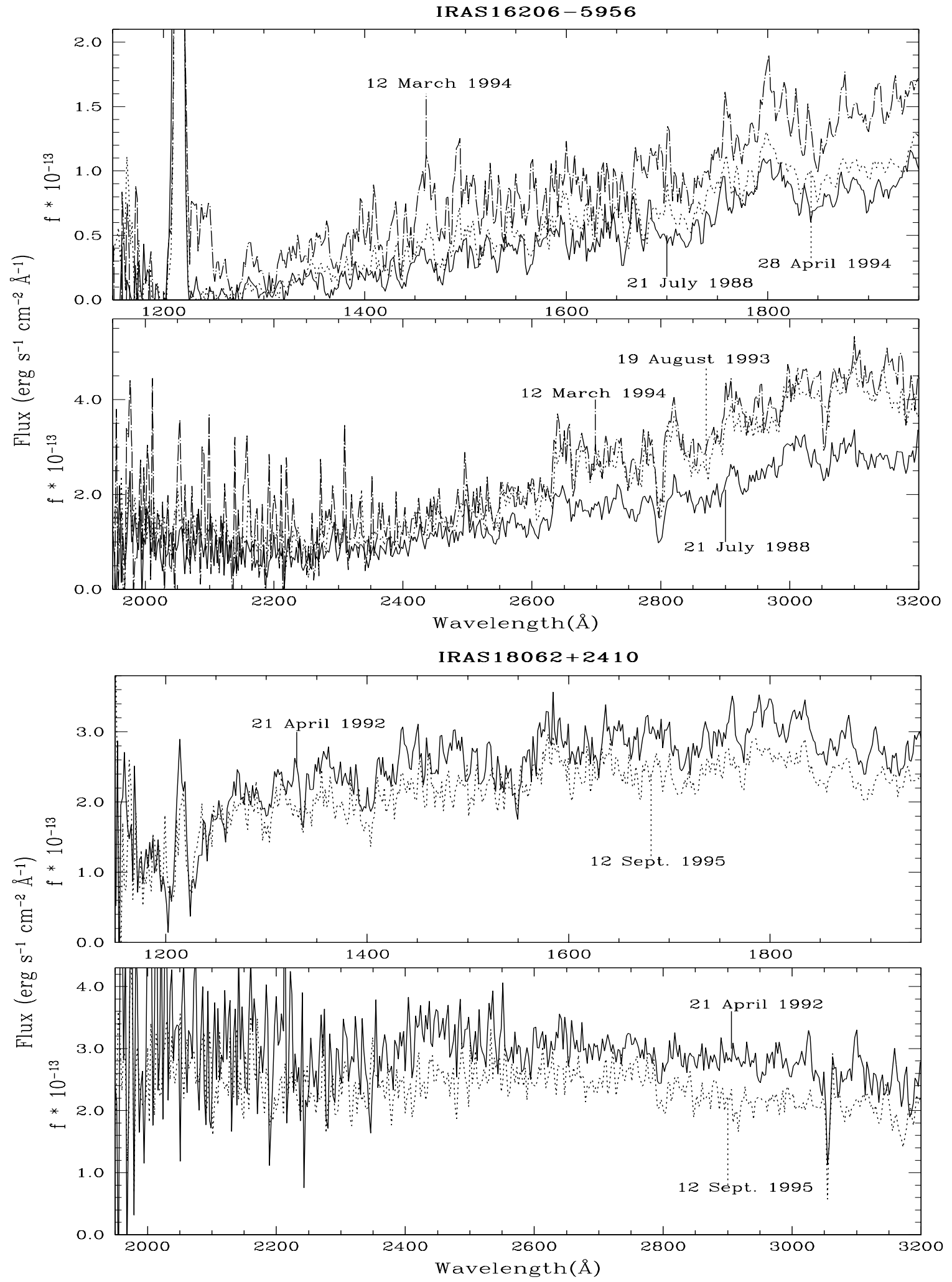

Fig. 1. The observed LWP and SWP spectra of IRAS 16206-5956 (SAO 243756) and IRAS 18062+2410 (SAO 85766) showing the variability.

For comparison, the flux of the standard stars were scaled in accordance with the difference between the $V$ magnitudes (after correcting for interstellar exintinction as determined from the $2200 \AA$ feature) of the standard star and the corresponding hot post-AGB candidate. 

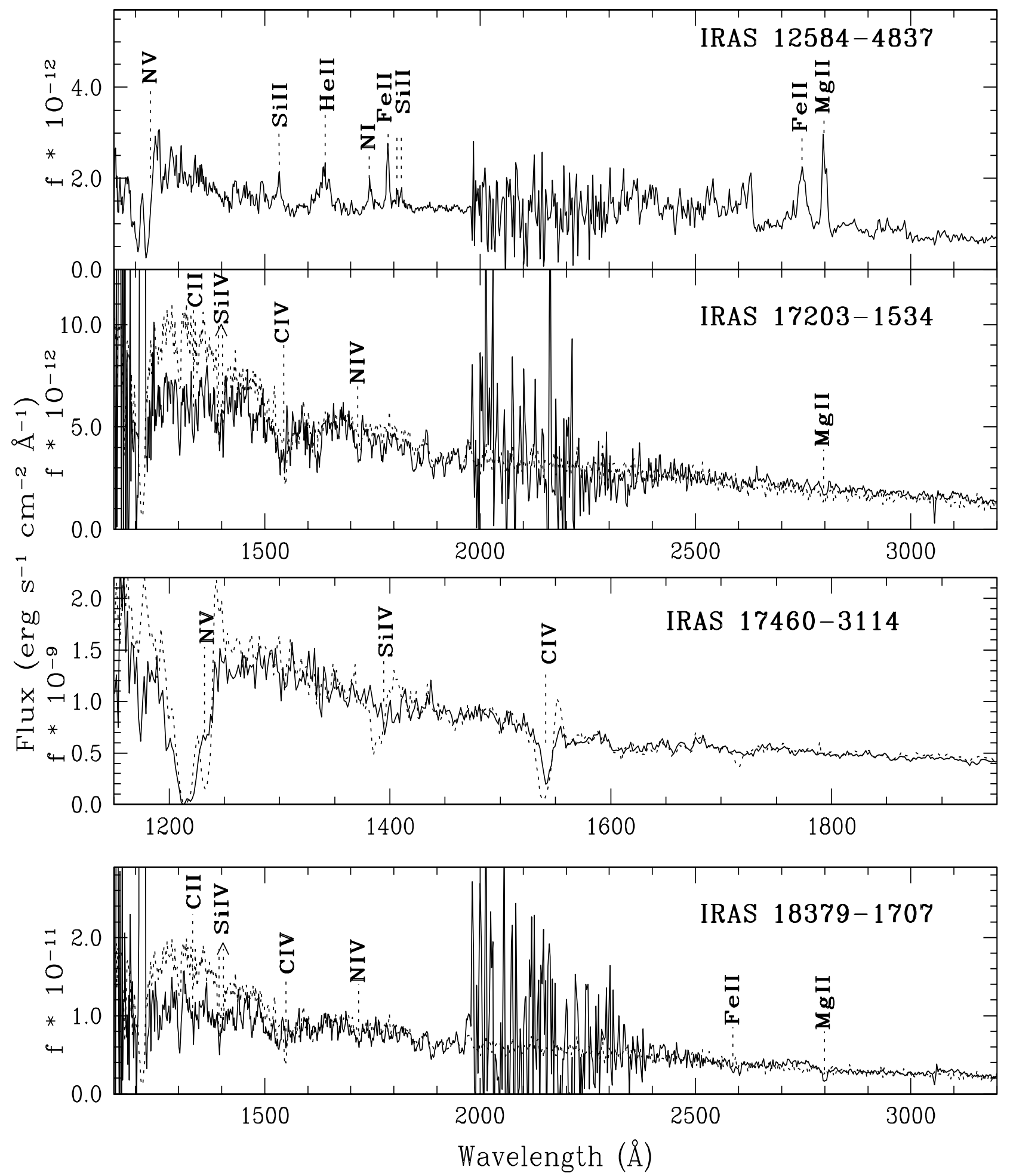

Fig. 2. The dereddened UV(IUE) spectra of hot post-AGB candidates with negligible circumstellar extinction (solid lines) compared with the dereddened UV(IUE) spectra of standard stars (dotted lines) from the atlas by Heck et al. (1984, Table 3). The spectra were dereddened using $E(B-V)$ estimated from the $2200 \AA$ feature in the UV. IRAS 17460-3114 was dereddened using $E(B-V)_{\text {total }}$ for the star. IRAS $12584-4837$ is a Be star in the optical.

\subsection{Spectral features in the UV}

The spectra from $1150 \AA$ to $3200 \AA$ A dereddened with $E(B-V)$ determined from the $2200 \AA$ feature are shown in Figs. 2, 3, 5a and $6 \mathrm{a}$. The complete Fig. 3 is available electronically at http://www.edpsciences.org. Lines of $\mathrm{NV}(1240 \AA)$, $\operatorname{CII}(1335 \AA), \quad \operatorname{SiIV}(1394, \quad 1403 \AA), \quad \operatorname{CIV}(1550 \AA)$, $\operatorname{NIV}(1718 \AA), \quad F e I I(2586-2631 \AA)$ and $\operatorname{MgII}(2800 \AA)$ typical of hot stars (Heck et al. 1984) and central stars of PNe were identified in the spectra of these stars. The UV line 


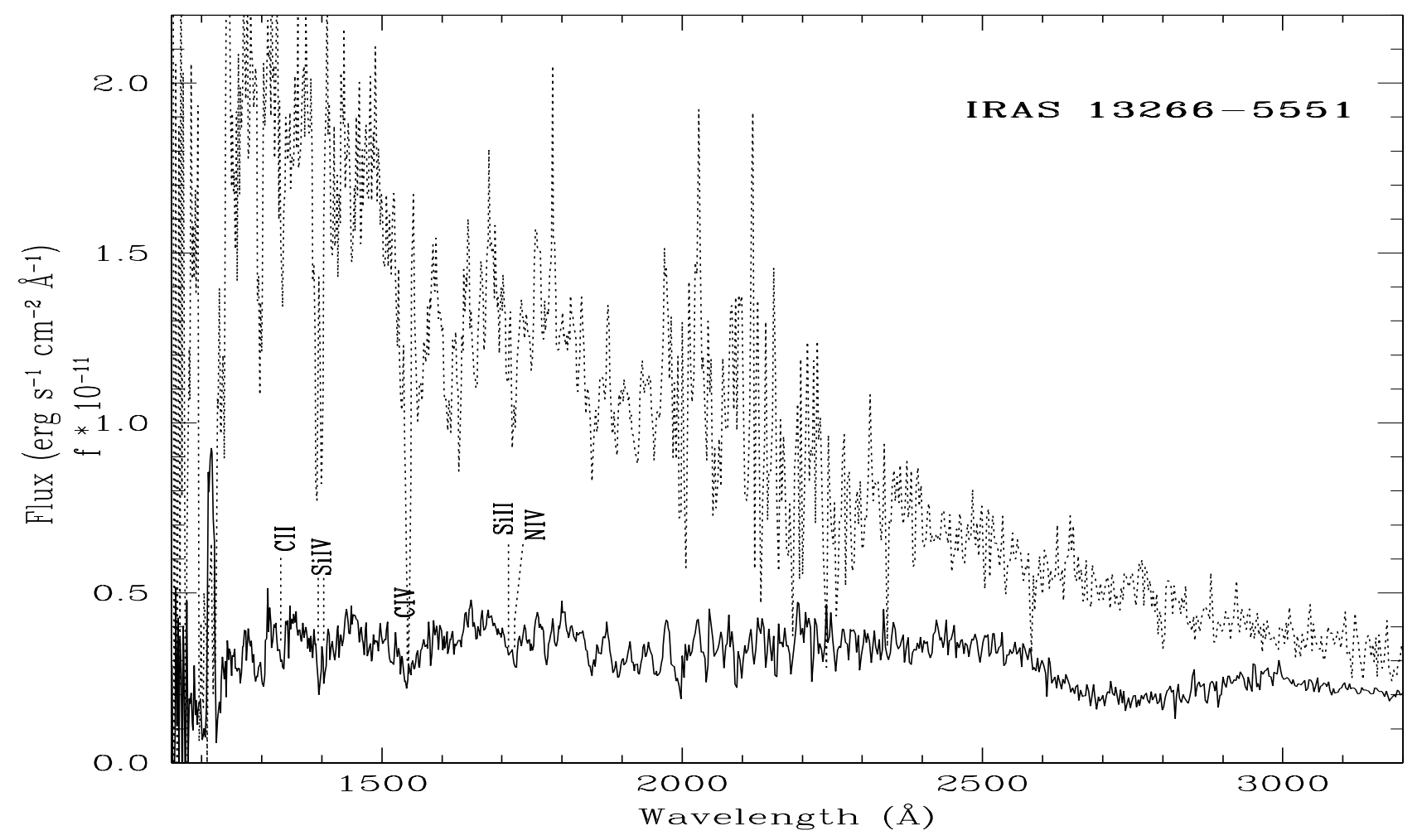

Fig. 3. The dereddened UV(IUE) spectra of hot post-AGB candidates (solid line) plotted alongwith the dereddened spectra of standard stars (dotted line) of similar optical spectral types from the atlas by Heck et al. (1984, Table 4a). The spectra have been dereddened using the $2200 \AA$ feature in the UV. Notice the reddened continua of the hot post-AGB candidates in comparison with the standard stars. The hot central star of IRAS 17423-1755 is not observed in the UV, possibly due to obscuration of the star by a dusty torus. The complete figure is available electronically at EDP Sciences.

strengths in the hot post-AGB candidates were compared with the line strengths in the standard stars. The absence of CIV in the B3-supergiant star, IRAS 14331-6435 (Hen3-1013) alongwith near normal line strengths of SiIV and NIV suggests the underabundance of carbon in this star. From the SiIV and CIV features it appears that IRAS 17074-1845 (Hen3-1347) and IRAS 17460-3114 (SAO 209306) may be slightly metal deficient. Based on its optical spectra, IRAS 18062+2410 (SAO 85766) was found to be metal poor and underabundant in carbon (Parthasarathy et al. 2000b; Mooney et al. 2002). In the UV also the line strengths appear to be weaker compared to a standard B1-supergiant. Metal deficiency has been observed in some high latitude hot post-AGB stars (see e.g. McCausland et al. 1992; Napiwotzki et al. 1994).

\subsection{Stars with negligible circumstellar extinction}

The UV continua and spectral features of IRAS 17203-1534, IRAS 17460-3114 (SAO 209306) and IRAS 18379-1707 (LSS 5112) were in good agreement with the dereddened UV(IUE) spectra of standard stars (Table 3 ) of similar optical spectral types (Fig. 2). The $E(B-V)$ values of these stars determined from the $2200 \AA$ feature are nearly the same as $E(B-V)_{\text {total }}$ (Table 3 ) suggesting negligible extinction of starlight due to circumstellar dust in these three cases. Emission lines of SiII(1533, 1808, $1817 \AA)$, HeII(1640 ̊), NI(1743 $)$ and FeII $(1785,2746 \AA)$ in the spectrum of IRAS 12584-4837 (Hen3-847) indicate the presence of hot plasma or a nebula.

\subsection{Stars with circumstellar extinction}

The UV(IUE) spectra of 10 stars (IRAS 13266-5551 (CPD-55 5588), IRAS 14331-6435 (Hen3-1013), IRAS 16202-5956 (SAO 243756), IRAS 17074-1845 (Hen3-1347), IRAS 173114924 (Hen3-1428), IRAS 18023-3409 (LSS 4634), IRAS $18062+2410$ (SAO 85766), IRAS 18371-3159 (LSE 63), IRAS 22023+5249 (LSIII +5224), IRAS 22495+5134 (LSIII +5142)), dereddened using the $2200 \AA$ feature in the UV, showed considerably reddened continua in comparison with the dereddened spectra of standard stars of similar optical spectral types (Figs. 3, 5 and 6). Comparing the interstellar extinction estimates from the $2200 \AA$ feature with the total extinction $\left(E(B-V)_{\text {total }}\right)$ towards these stars, we find that these stars have considerable UV deficiency and circumstellar extinction. The hot central star of the bipolar proto-planetary nebula (PPN), IRAS 17423-1755 (Hen3-1475) was not detected in a 35 min exposure with the SWP camera. This may be due to obscuration of the central star by a dusty disk. HST WFPC2 images of the object showed the presence of a dusty torus with a spatial extent of $2^{\prime \prime}$ (Borkowski et al. 1997). 
Table 3. Hot post-AGB candidates with negligible circumstellar extinction.

\begin{tabular}{cccccccccc}
\hline \hline IRAS & $V$ & $(B-V)_{\text {obs }}$ & $\begin{array}{c}E(B-V) \\
\text { I.S. }\end{array}$ & $\begin{array}{c}E(B-V) \\
(2200 \AA)\end{array}$ & $\begin{array}{c}\text { Sp. type } \\
\text { Optical }\end{array}$ & $\begin{array}{c}E(B-V) \\
\text { total }\end{array}$ & $\begin{array}{c}\text { Standard } \\
\text { star }\end{array}$ & $\begin{array}{c}\text { Sp. type } \\
\text { std. star }\end{array}$ & $\begin{array}{c}E(B-V) \\
\text { std. star }\end{array}$ \\
\hline $12584-4837$ & $10.58^{a}$ & $0.07^{a}$ & 0.18 & 0.25 & $\mathrm{Be}^{1}$ & - & - & - & - \\
$17203-1534$ & $12.02^{a}$ & $0.35^{a}$ & 0.44 & 0.56 & B1IIIpe & 0.61 & HD 173502 & B1II & 0.09 \\
$17460-3114$ & $7.94^{b}$ & $0.23^{b}$ & - & 0.54 & O8III & 0.54 & HD 162978 & O7.5II & 0.35 \\
$18379-1707$ & $11.93^{b}$ & $0.45^{b}$ & - & 0.70 & B1IIIpe & 0.71 & HD 173502 & B1II & 0.11 \\
\hline
\end{tabular}

Photometry is from: ${ }^{a} \mathrm{Hog}$ et al. (2000); ${ }^{b}$ Reed (1998). Spectral types of the hot post-AGB candidates are from Parthasarathy et al. (2000a) except ${ }^{1}$ Kazarovets et al. (2000).

Table 4a. Hot post-AGB candidates with observable circumstellar extinction.

\begin{tabular}{ccccccccccc}
\hline \hline IRAS & $V$ & $(B-V)_{\text {obs }}$ & $\begin{array}{c}E(B-V) \\
\text { I.S. }\end{array}$ & $\begin{array}{c}E(B-V) \\
(2200 \AA)\end{array}$ & $\begin{array}{c}\text { Sp. type } \\
\text { Optical }\end{array}$ & $\begin{array}{c}E(B-V) \\
\text { total }\end{array}$ & $\begin{array}{c}\text { Standard } \\
\text { star }\end{array}$ & $\begin{array}{c}\text { Sp. type } \\
\text { std. star }\end{array}$ & $\begin{array}{c}E(B-V) \\
\text { std. star }\end{array}$ & $\begin{array}{c}E(B-V) \\
\text { C.S. }\end{array}$ \\
\hline $13266-5551$ & $10.68^{b}$ & $0.31^{b}$ & 0.53 & 0.38 & B1Ibe & 0.51 & HD 77581 & B0.5Ia & 0.77 & 0.15 \\
$14331-6435$ & $10.90^{b}$ & $0.58^{b}$ & - & 0.44 & B3Ie & 0.71 & HD 198478 & B3Ia & 0.58 & 0.26 \\
$17074-1845$ & $11.47^{a}$ & $0.46^{a}$ & 0.28 & 0.24 & B3IIIe & 0.66 & HD 51309 & B3II & 0.13 & 0.39 \\
$17311-4924$ & $10.68^{c}$ & $0.40^{c}$ & 0.22 & 0.28 & B1IIe & 0.66 & HD 51283 & B2III & 0.02 & 0.39 \\
$17423-1755$ & $12.64^{d}$ & $0.66^{d}$ & 0.67 & - & Be & - & - & - & - & - \\
$18023-3409$ & $11.55^{b}$ & $0.46^{b}$ & 0.44 & 0.30 & B2IIIe & 0.70 & HD 51283 & B2III & 0.02 & 0.43 \\
$18371-3159$ & $11.98^{a}$ & $0.11^{a}$ & 0.15 & 0.13 & B1Iabe & 0.30 & HD 122879 & B0Ia & 0.42 & 0.11 \\
$22023+5249$ & $12.52^{a}$ & $0.69^{a}$ & - & 0.37 & B ${ }^{1}$ & - & HD 41117 & B2Ia & 0.52 & 0.44 \\
$22495+5134$ & $11.78^{a}$ & $0.22^{a}$ & 0.357 & 0.33 & PN $^{2}$ & - & HD 38666 & 09V & 0.02 & 0.20 \\
\hline
\end{tabular}

Photometry is from: ${ }^{a} \mathrm{Hog}$ et al. (2000); ${ }^{b}$ Reed (1998); ${ }^{c}$ Kozok (1985a); ${ }^{d}$ Gauba et al. (2003).

Spectral types of the hot post-AGB candidates are from Parthasarathy et al. (2000a) except ${ }^{1}$ Simbad database; ${ }^{2}$ Acker et al. (1992).

Table 4b. Hot post-AGB candidates showing variation in the UV.

\begin{tabular}{|c|c|c|c|c|c|c|c|c|c|c|}
\hline IRAS & $\begin{array}{c}V \\
\text { mag }\end{array}$ & $(B-V)_{\mathrm{obs}}$ & $\begin{array}{c}E(B-V) \\
\text { I.S. }\end{array}$ & $\begin{array}{l}E(B-V) \\
(2200 \AA)\end{array}$ & $\begin{array}{l}\text { Sp. type } \\
\text { Optical }\end{array}$ & $\begin{array}{c}E(B-V) \\
\text { total }\end{array}$ & $\begin{array}{c}\text { Standard } \\
\text { star }\end{array}$ & $\begin{array}{l}\text { Sp. type } \\
\text { std. star }\end{array}$ & $\begin{array}{c}E(B-V) \\
\text { std. star }\end{array}$ & $\begin{array}{c}E(B-V) \\
\text { C.S. }\end{array}$ \\
\hline \multirow[t]{2}{*}{$16206-5956$} & \multirow[t]{2}{*}{$9.76^{a}$} & \multirow[t]{2}{*}{$0.31^{a}$} & \multirow[t]{2}{*}{0.22} & \multirow[t]{2}{*}{0.13} & \multirow[t]{2}{*}{$\mathrm{A} 0 \mathrm{Ia}^{1}$} & \multirow[t]{2}{*}{0.29} & \multirow[t]{2}{*}{ HD 21389} & \multirow[t]{2}{*}{ A0Ia } & \multirow[t]{2}{*}{0.79} & $\begin{array}{c}0.54 \\
(21 / 07 / 88)\end{array}$ \\
\hline & & & & & & & & & & $\begin{array}{c}0.24 \\
(12 / 03 / 94)\end{array}$ \\
\hline \multirow[t]{2}{*}{$18062+2410$} & \multirow[t]{2}{*}{$11.54^{b}$} & \multirow[t]{2}{*}{$0.05^{b}$} & \multirow[t]{2}{*}{0.11} & \multirow[t]{2}{*}{0.08} & \multirow[t]{2}{*}{$\mathrm{B} 1 \mathrm{I}^{2}$} & \multirow[t]{2}{*}{0.24} & \multirow[t]{2}{*}{-} & \multirow[t]{2}{*}{-} & \multirow[t]{2}{*}{-} & $\begin{array}{c}0.03 \\
(21 / 04 / 92)\end{array}$ \\
\hline & & & & & & & & & & $\begin{array}{c}0.30 \\
(12 / 09 / 95)\end{array}$ \\
\hline
\end{tabular}

Photometry is from: ${ }^{a}$ Reed (1998); ${ }^{b}$ Arkhipova et al. (1999).

Spectral types are from: ${ }^{1}$ Schild et al. (1983); ${ }^{2}$ Parthasarathy et al. (2000b).

\subsubsection{Modelling the circumstellar extinction}

To account for the observed UV deficiency in the 10 hot postAGB candidates mentioned in Sect. 3.3 and to understand the shape of the UV continuum in these stars, we investigated the circumstellar extinction law in these cases. Waters et al. (1989) modelled the circumstellar extinction in the case of the postAGB star, HR 4049. We followed the same procedure here.

We plotted the logarithmic difference between the dereddened UV flux of a hot post-AGB candidate (normalised to its $V$-band flux) and the dereddened UV flux of the corresponding standard star (normalised to its $V$-band flux), i.e. $\Delta=\log \left(f_{\lambda} / f_{\mathrm{v}}\right)_{\text {star }}-\log \left(f_{\lambda} / f_{\mathrm{v}}\right)_{\text {standard }}$ vs. $\lambda^{-1}$.

Figure 4 shows the plot of the logarithmic flux deficiency due to circumstellar dust from 3.2 to $8 \mu^{-1}$ for the 10 stars. The complete Fig. 4 is available electronically at http://www . edpsciences.org. Best fit lines were obtained by minimising the chi-square error statistic. E.g., in the case of IRAS 17311-4924 (Hen3-1428), we obtained, $\Delta=0.07-$ $0.10 \lambda^{-1}$. To derive the circumstellar extinction $\left(E(B-V)_{\text {C.S. }}\right)$ in magnitudes (Tables $4 \mathrm{a}, \mathrm{b}) \Delta$ had to be multiplied by -2.5 and $\lambda=0.44 \mu$ was used. 


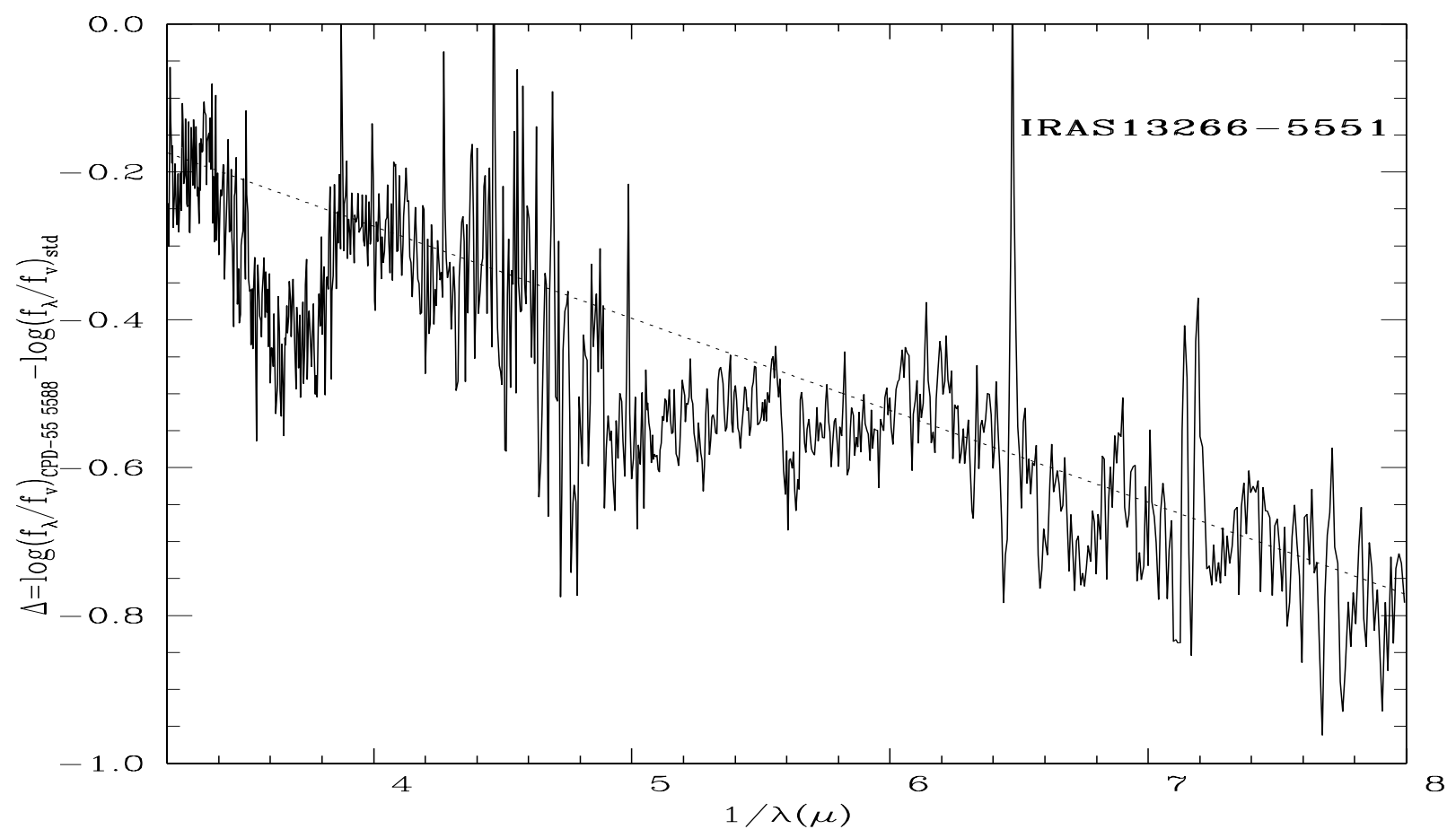

Fig. 4. 10 hot post-AGB candidates showed a pronounced UV deficiency when compared with standard stars of similar optical spectral types. Here, we have plotted the logarithmic flux deficiency of these stars in the UV from 3.2 to $8 \mu^{-1}$. The straight line fits are obtained by minimising the chi-square statistic. We find that the flux deficiency in the UV is proportional to $\lambda^{-1}$. The bump at $\sim 3.7 \mu^{-1}$ in the plot of IRAS $13266-5551$ is due to the saturated LWP spectrum of the star. This region was not used in obtaining a fit. Similarly, for IRAS 18023-3409, the LWP spectrum appears to have saturation effects. For IRAS $18371-3159$, the bump from $\sim 5$ to $5.6 \mu^{-1}$ is observed because of spurious broad absorption features from $1800 \AA$ to $1950 \AA$ in the SWP spectrum of the star. The complete figure is available electronically at EDP Sciences.

For IRAS $22023+5249$ (LSIII +5224), only a B spectral type is listed in literature. We compared the spectrum of this star with that of a B2-supergiant standard star. IRAS $22495+5134$ (LSIII +5142) was detected as a PN with an angular extent of 4" (Acker et al. 1992) Central stars of $\mathrm{PNe}$ have temperatures in excess of $\sim 30000 \mathrm{~K}$ corresponding to spectral types of $\mathrm{O} 9$ or hotter. We compared the UV(IUE) spectra of this star with a standard O9V star (HD 38666).

For each of the 10 hot post-AGB candidates we found that the circumstellar extinction varies as $\lambda^{-1}$ (Fig. 4). The derived $E(B-V)_{\text {C.S. }}$ values in Table $4 \mathrm{a}$ account well for the difference between the total and the interstellar extinction (from the $2200 \AA$ feature) values. $E(B-V)_{\text {C.S. values in Table } 4 \mathrm{~b} \text { are in }}$ excess of the difference between $E(B-V)_{\text {total }}$ and the interstellar extinction from $2200 \AA$. This may be because of the variable nature of these stars and because the $V$ and $(B-V)$ magnitudes at each epoch of the IUE observations are not known. Mean $V$ and $(B-V)$ magnitudes from literature have been used for each of these two stars (see Sect. 3.3.2 below).

\subsubsection{Variations in the UV(IUE) spectra of IRAS 16206-5956 (SAO 243756) and IRAS 18062+2410 (SAO 85766)}

IRAS 16206-5956 was found to be variable in the UV (Fig. 1). The spectrum of the star has changed from 21 July 1988 to 12 March 1994 and from 12 March 1994 to 28 April 1994 suggesting both long term and short term variability. The maximum flux in the UV was observed on 12 March 1994 Schild et al. (1983) found it to be variable in the optical with $\Delta V=0.13$. Since the $V$ magnitudes of the star at the epochs of the UV(IUE) observations are not known, we used a mean $V$ magnitude (=9.76) from the photometric and spectroscopic database for Stephenson-Sanduleak Luminous Stars in the Southern Milky Way (Reed 1998). Its spectral type in literature is listed as A3Iabe (Humphreys 1975; Parthasarathy et al. 2000a) and A0Iae (Schild et al. 1983; Garrison et al. 1977). Oudmaijer (1996) listed it as B8Ia, citing the Simbad database. However, we could not find a reference for the same. We compared the dereddened IUE spectra of the star at different epochs with the dereddened spectra of A3Ib (HD 104035) and A0Ia (HD 21389) standard stars (Fig. 5a). A match could not be obtained in either case. Finally, we adopted the A0Ia standard star (HD 21389) and modelled the circumstellar extinction in the case of SAO 243756 (as outlined in Sect. 3.3.1) for the spectra taken on 12 March 1994 (maximum observed IUE flux) and 21 July 1988 (minimum observed IUE flux). The circumstellar extinction was found to be linear in $\lambda^{-1}$ at both epochs (Fig. 5b, Table 4b).

The UV flux from IRAS $18062+2410$ has decreased in Sep. 1995, compared to the flux from the hot central star in April, 1992 (Fig. 1). From an analysis of the high resolution optical spectra of the star, Parthasarathy et al. (2000b) and Mooney et al. (2002), found it to be metalpoor $([\mathrm{M} / \mathrm{H}] \sim-0.6)$. Since there are no hot (OB-spectral types), metal-poor standard stars in the UV, we compared the 

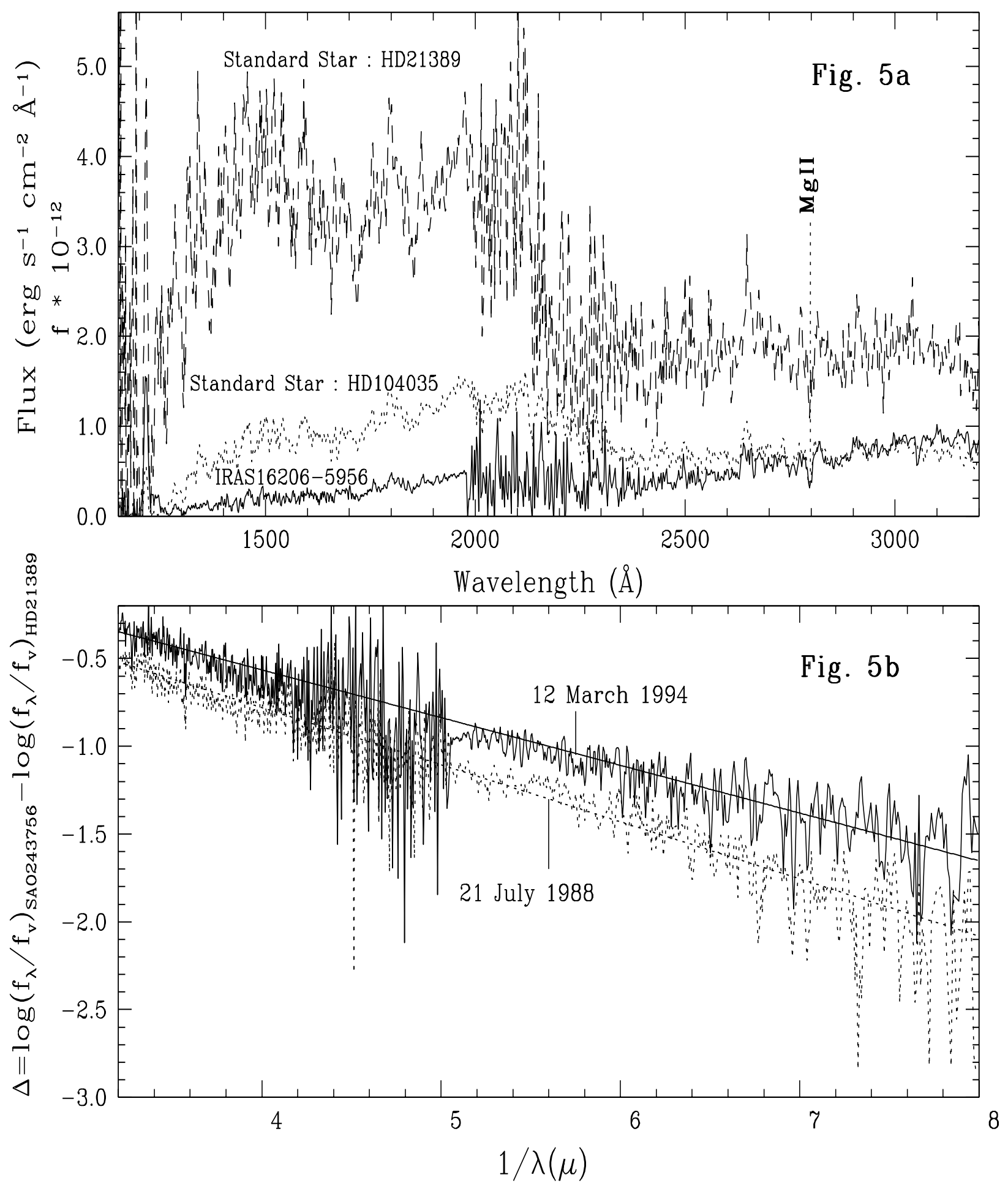

Fig. 5. IRAS 16206-5956 (SAO 243756) and the standard A3Ib (HD 104035) and A0Ia (HD 166937) stars were dereddened using the $2200 \AA$ feature in the UV. Figure 5a shows the dereddened 12 March 1994 spectrum of SAO 243756 (solid line) alongwith the dereddened spectra of the standard A0Ia (dash line) and A3Ib stars (dotted line). SAO 243756 is fainter in the spectrum taken on 21 July 1988 (Ref. Fig. 1). Adopting the A0Ia standard star, Fig. 5b shows the modelled circumstellar extinction for the spectrum on 12 March 1994 (solid line) and 21 July 1988 (dotted line) respectively.

UV spectrum of this star with a Kurucz model closest to the effective temperature, gravity and metallicity of the star in the optical (Parthasarathy et al. 2000b; Mooney et al. 2002) We adopted $T_{\text {eff }}=23000 \mathrm{~K}, \log g=3.0$ and $[\mathrm{M} / \mathrm{H}]=-0.5$ (Fig. 6a). Arkhipova et al. $(1999,2000)$ found that it shows irregular rapid light variations in the optical with an amplitude of upto 0.3 in $V$. We adopted mean $V=11.54$ (Arkhipova et al. 1999). Following the prescription in Sect. 3.3.1, we find that the circumstellar extinction varies as $\lambda^{-1}$ (Fig. 6b, Table 4b) and has increased in magnitude from 1992 to 1995. 


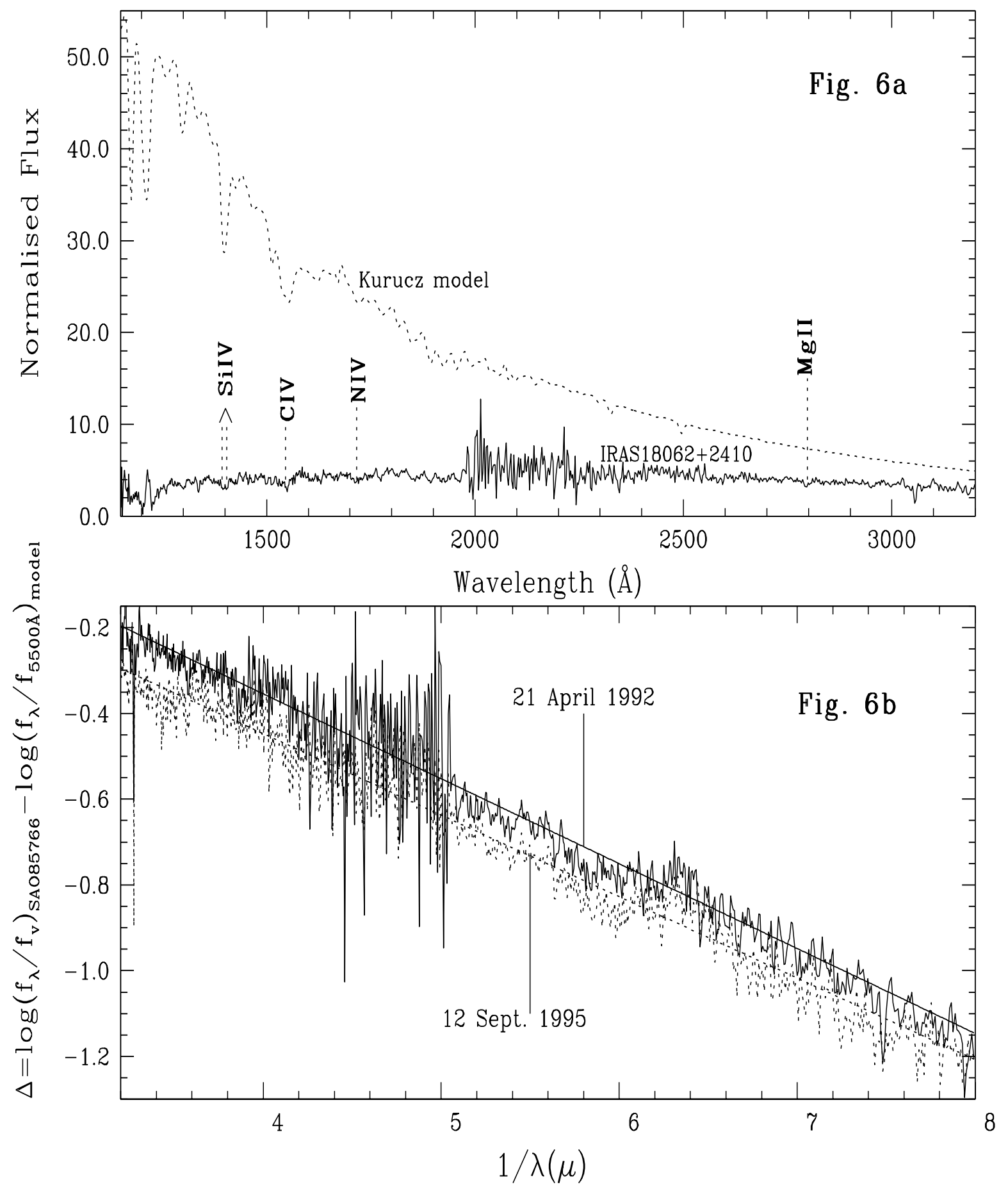

Fig. 6. IRAS $18062+2410$ (SAO 85766) was dereddened using the $2200 \AA$ feature in the UV. Figure 6a shows the dereddened 1992 spectrum of SAO 85766 normalised to its $V$-band flux (solid line) alongwith Kurucz model (dotted line) of $T_{\text {eff }}=23000 \mathrm{~K}, \log g=3.0,[\mathrm{M} / \mathrm{H}]=-0.5$ normalised to the flux at $5500 \AA$ A. SAO 85766 has become fainter in the 1995 spectrum of the star. Figure $6 \mathrm{~b}$ shows the modelled circumstellar extinction for 1992 (solid line) and 1995 (dotted line).

\subsection{Central star parameters and energy budget}

For the 3 hot post-AGB candidates with negligible circumstellar extinction, IRAS 17203-1534, IRAS 17460-3114 (SAO 209306) and IRAS 18379-1707 (LSS 5112), we modelled the spectra using solar metallicity Kurucz (1994) model atmospheres (Fig. 7) and derived the effective temperatures and gravities of these stars. The $V$-band fluxes of the stars were corrected for extinction assuming the normal interstellar extinction law, i.e. $A_{\mathrm{v}}=3.1 \times E(B-V)_{2200}$ (see e.g. Seaton 1979). The stellar flux distributions normalised to the corrected $V$-band flux of each star were compared with Kurucz models normalised to the respective model's flux at $5500 \AA$. $\log g$ in the case of the O8III star, IRAS 17460-3114 (SAO 209206) was estimated to be 4.0. This value is uncertain due to the non-availability of Kurucz models of lower gravity at 
Table 5. Central star parameters and Energy budget.

\begin{tabular}{|c|c|c|c|c|c|c|c|}
\hline Star No. & IRAS & $T_{\text {eff }}(\mathrm{K})$ & $\log g$ & $\begin{array}{c}F_{\text {fir }} \\
\times 10^{-12} \mathrm{Wm}^{-2}\end{array}$ & $\begin{array}{c}F_{\text {star }} \\
\times 10^{-12} \mathrm{Wm}^{-2}\end{array}$ & $\overline{F_{\text {fir }} / F_{\text {star }}}$ & $\begin{array}{l}\text { Wind velocity } \\
\mathrm{km} \mathrm{s}^{-1}\end{array}$ \\
\hline 1. & $12584-4837$ & - & - & 10.90 & 6.07 & 1.79 & -3769 \\
\hline 2. & $13266-5551$ & 20800 & 2.8 & 5.41 & 9.29 & 0.58 & -1821 \\
\hline 3. & 14331-6435 & 16200 & 2.6 & 15.40 & 4.50 & 3.42 & - \\
\hline \multirow[t]{2}{*}{4.} & $16206-5956$ & 11200 & 2.3 & 1.73 & $2.61(21 / 07 / 88)$ & 0.66 & - \\
\hline & & & & & $2.97(12 / 03 / 94)$ & 0.58 & - \\
\hline 5. & $17074-1845$ & 17100 & 3.4 & 1.67 & 1.44 & 1.16 & - \\
\hline 6. & $17203-1534^{1}$ & $19000 \pm 1000$ & $2.5 \pm 0.5$ & 1.51 & 8.86 & 0.17 & -2402 \\
\hline 7. & $17311-4924$ & 20300 & 3.0 & 21.80 & 4.01 & 5.44 & -1066 \\
\hline 8. & 17423-1755 & - & - & 6.41 & 1.17 & 5.48 & - \\
\hline 9. & $17460-3114^{1}$ & $35000 \pm 2500$ & $4.0 \pm 1.0$ & 4.95 & 876.8 & 0.006 & -1463 \\
\hline 10. & 18023-3409 & 20300 & 3.0 & 0.58 & 1.13 & 0.51 & -2048 \\
\hline \multirow[t]{2}{*}{11.} & $18062+2410^{2}$ & 23000 & 2.6 & 2.90 & $1.64(21 / 04 / 92)$ & 1.77 & - \\
\hline & & & & & $1.33(12 / 09 / 95)$ & 2.18 & - \\
\hline 12. & 18371-3159 & 20800 & 2.9 & 0.93 & 2.17 & 0.43 & - \\
\hline 13. & $18379-1707^{1}$ & $19000 \pm 1000$ & $2.5 \pm 0.5$ & 3.20 & 16.96 & 0.19 & - \\
\hline 14. & $22023+5249$ & - & - & 3.45 & 1.14 & 3.03 & -3978 \\
\hline 15. & $22495+5134$ & - & - & 1.74 & 3.72 & 0.47 & - \\
\hline
\end{tabular}

${ }^{1}$ The effective temperatures and gravities were estimated using solar metallicity Kurucz (1994) models.

${ }^{2}$ Effective temperature and gravity are from Mooney et al. (2002).

the high temperature $\left(T_{\text {eff }}=35000 \mathrm{~K}\right)$ of the star. The star may also be slightly metal deficient as discussed in Sect. 3.1. For the remaining stars, we obtained the effective temperatures and gravities based on their optical spectral types (Lang 1992). Table 5 lists the adopted $T_{\text {eff }}$ and $\log g$ values. The $T_{\text {eff }}$ and $\log g$ values estimated from the optical spectral types of the stars may be uncertain by $\sim \pm 1000 \mathrm{~K}$ and \pm 1.0 respectively. High resolution optical spectra of these stars are required for an accurate determination of $T_{\text {eff }}$ and $\log g$. The stars were placed on Schönberber's $(1983,1987)$ post-AGB evolutionary tracks for core masses $\left(M_{\mathrm{c}}\right)$ of $0.546,0.565,0.598$ and $0.644 M_{\odot}$ (Fig. 8).

The far-IR flux distributions of the hot post-AGB candidates must necessarily be due to flux from the hot central stars absorbed and re-radiated by the cold circumstellar dust envelopes which are a remnant of mass-loss on the AGB phase of the star. Hence, the integrated far-IR flux $\left(F_{\text {fir }}\right)$ must be comparable to or less than the integrated stellar flux $\left(F_{\text {star }}\right)$. We estimated $F_{\text {fir }}$ from the $12 \mu$ to $100 \mu$ IRAS flux distributions for the stars. The integrated stellar flux $\left(F_{\text {star }}\right)$ from $1150 \AA$ to $5500 \AA$ was estimated by combining the IUE spectra with the $U, B, V$ magnitudes of the stars from literature. The IUE spectra and the $U, B, V$ magnitudes were corrected for interstellar extinction derived from the $2200 \AA$ feature. IRAS $17423-$ 1755 was not detected in the UV. Its $U, B, V, R, I$ magnitudes (Gauba et al. 2003) were corrected for interstellar extinction using the standard extinction law (Rieke \& Lebofsky 1985) and the integrated stellar flux from $3650 \AA$ ( $U$-band) to $9000 \AA$ (I-band) was estimated. In the three cases with negligible circumstellar extinction (IRAS 17203-1534, IRAS 17460-3114 and IRAS 18379-1707), $F_{\text {fir }}$ was comparable to or signifi-

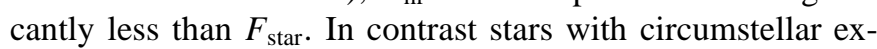
tinction in the UV, e.g. IRAS 14331-6435, IRAS 17074-1845, IRAS 17311-4924, IRAS 17423-1755, IRAS 18062+2410 and IRAS $22023+5249$ ) showed a high ratio of $F_{\text {fir }} / F_{\text {star }}$ indicating partial obscuration of the central stars. These stars may have dusty circumstellar disks.

\subsection{Terminal wind velocity}

The wavelength at which the shortward edge of the CIV (or NV) absorption profile intersects the stellar continuum is usually used to estimate the terminal wind velocities $\left(v_{\infty}\right)$ of hot stars from low resolution IUE spectra. However, this value is usually an upper limit to the true $v_{\infty}$. Alternatively, the difference between the absorption and emission line centers $(\Delta v)$ are used to estimate $v_{\infty}$ from low resolution IUE spectra (Prinja 1994). But high dispersion studies (see e.g. Perinotto et al. 1982) have shown that $\Delta v$ is less than $v_{\infty}$.

Considerable circumstellar extinction in 10 of the 15 stars discussed in this paper, has significantly distorted the continuum flux distributions and line intensities of the CIV profiles. Hence, the point where the blue absorption edge of the CIV profile intersects the UV continuum is not reliable. These factors also contribute to the fact that the CIV P-Cygni profiles are not clearly observed in the low resolution spectra of these stars. The emission peaks may be too weak or lost in the reddened continuum. Hence it is not possible to estimate $v_{\infty}$ from the shortward edge of the CIV absorption profile or from the difference between the absorption and emission line centers.

The CIV resonance doublet $(1548 \AA, 1550 \AA$ ) is not resolved in the IUE low resolution spectra. Stellar wind may be assumed to be absent in a B0V star. We measured the absorption minimum of the CIV feature in the UV(IUE) spectrum of a B0V standard star, HD 36512 (1548.4 $\AA$ ) from the atlas by Heck et al. (1984). The difference between this wavelength and the absorption minima of the CIV feature in the IUE spectra of our hot post-AGB candidates was used to estimate the wind velocities in these stars. If instead, we had used the mean 

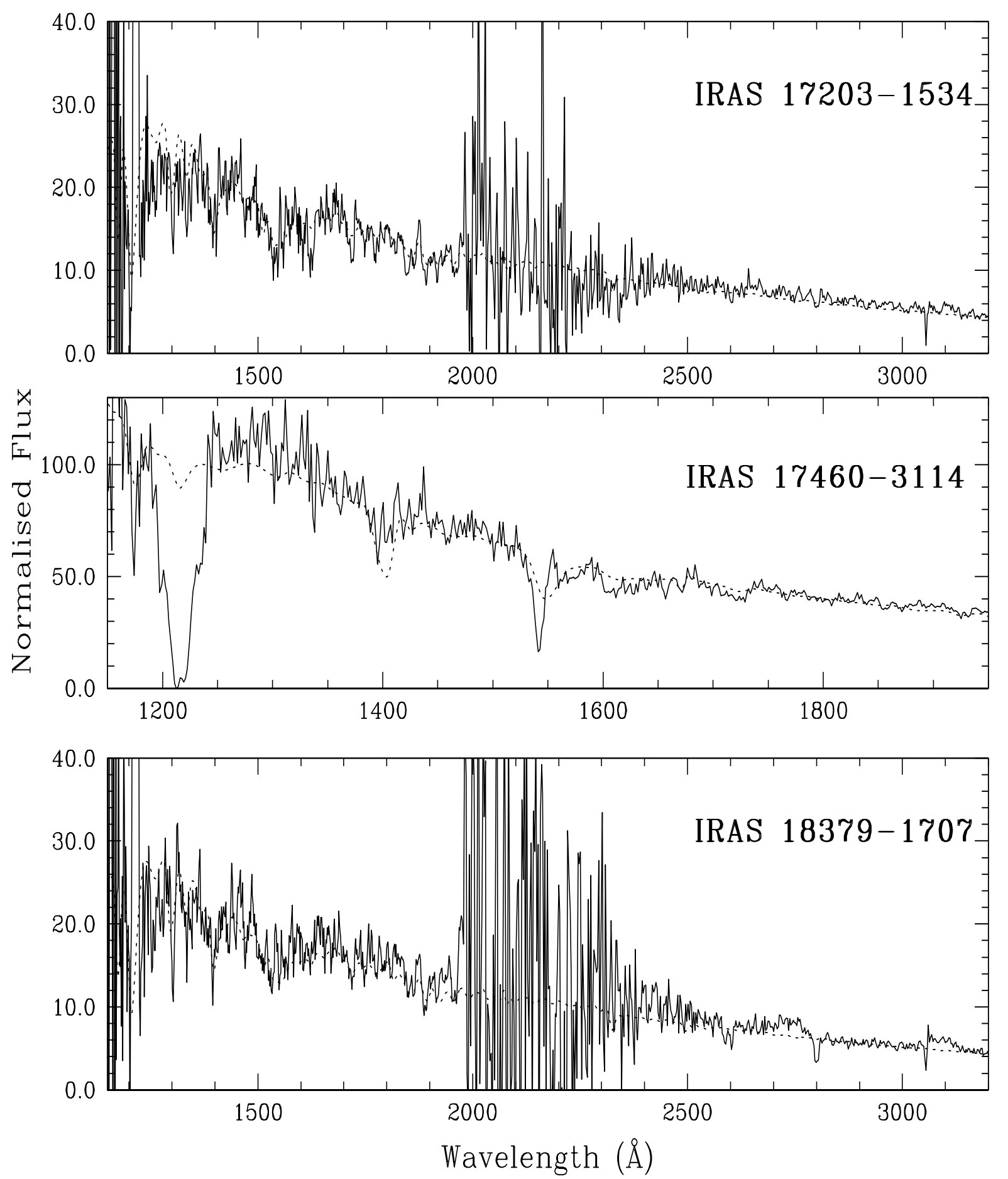

Fig. 7. The dereddened UV(IUE) spectra of hot post-AGB candidates with negligible circumstellar extinction (solid line), normalised to their $V$-band fluxes are plotted alongwith solar metallicity Kurucz (1994) model spectra (dotted line), normalised to each model's flux at $5500 \AA$.

laboratory wavelength of the CIV resonance doublet (1549 ̊), the estimated terminal wind velocites would have been greater by $116 \mathrm{~km} \mathrm{~s}^{-1}$. The NV resonance doublet (1238 $\mathrm{\AA}, 1242 \AA$ ) is also unresolved in low resolution IUE spectra. Moreover, the NV feature is often contaminated by geocoronal Lyman $\alpha$. From the standard star atlas (Heck et al. 1984) we were unable to find a star in which the NV line is distinguishable from Lyman $\alpha$ and unaffected by stellar wind. Hence, in the case of IRAS 12584-4837, we estimated the wind velocity from the difference between the NV absorption minimum and the mean laboratory wavelength of the line (1240 ̊). The estimated wind velocities are listed in Table 5. No shift was observed in the case of IRAS 17074-1845, IRAS 18062+2410, IRAS 18379-1707 and IRAS 22495+5134. CIV (or NV) lines were not observed in IRAS 14331-6435 and IRAS 162065958. The SiIV (1394 A) absorption line in IRAS 14331-6435 did not show a wavelength shift due to stellar wind. For low resolution IUE spectra, the FWHM of the instrumental width is 


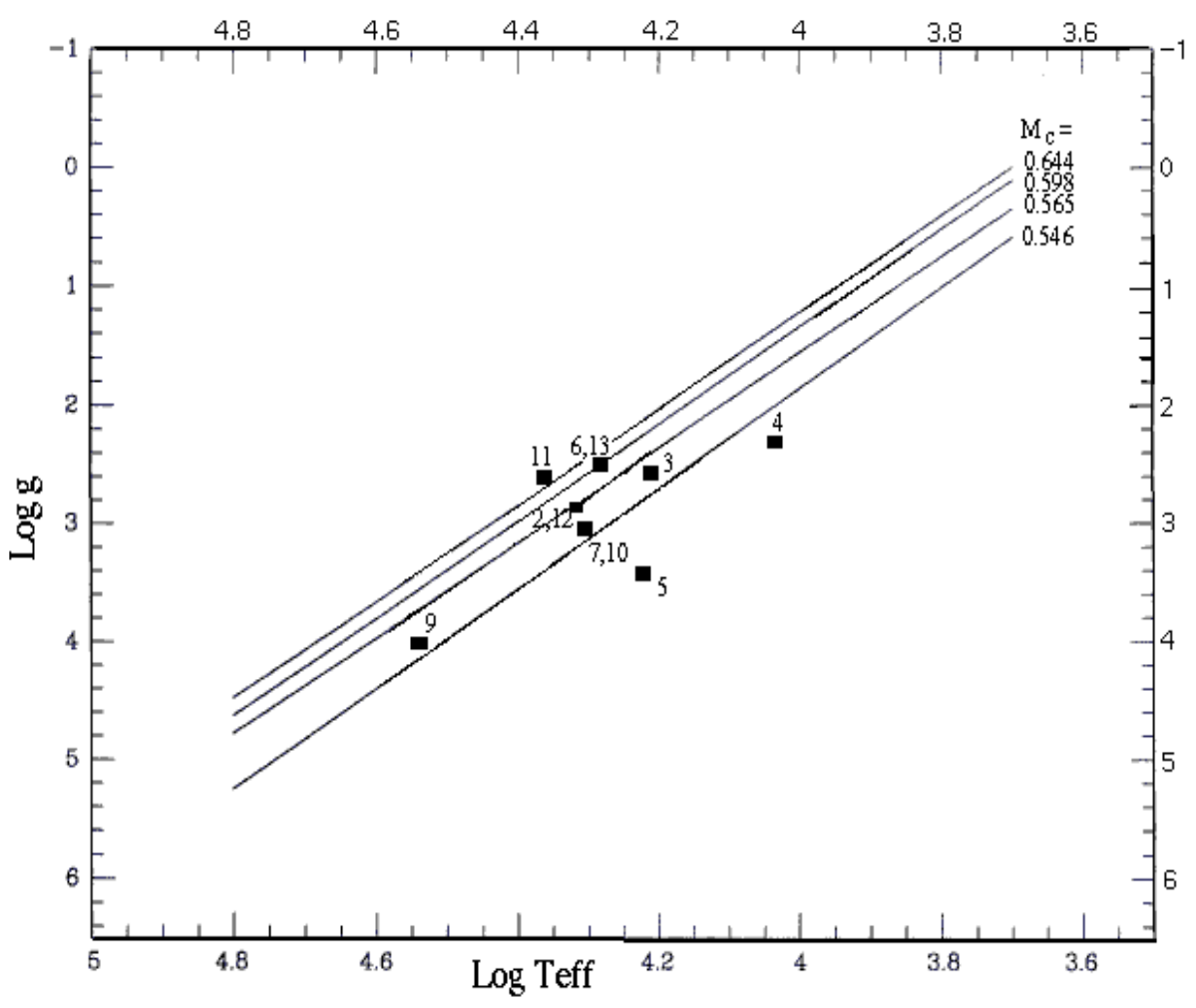

Fig. 8. Positions of the hot post-AGB candidates on the $\log g-\log T_{\text {eff }}$ diagram showing the post-AGB evolutionary tracks of Schönberner $(1983,1987)$ for core masses of $0.546,0.565,0.598$ and $0.644 M_{\odot}$.

$5 \AA$ A (Castella \& Barbero 1983). Assuming a $3 \AA$ measurement error in estimating the blue shift of the lines from our low resolution spectra, would correspond to an error of $\sim 580 \mathrm{~km} \mathrm{~s}^{-1}$ and $726 \mathrm{~km} \mathrm{~s}^{-1}$ in the estimate of wind velocites from CIV and NV respectively. However, our terminal wind velocities exceeded this error estimate. So, we may conclude that although a crude estimate is obtained of the terminal wind velocities, it nevertheless indicates the presence of stellar winds and post-AGB mass-loss in some of these stars.

\section{Discussion and conclusions}

We analysed the UV(IUE) spectra of 15 hot post-AGB candidates. In 11 cases (IRAS 13266-5551 (CPD-55 5588), IRAS 14331-6435 (Hen3-1013), IRAS 16206-5956 (SAO 243756), IRAS 17074-1845 (Hen3-1347), IRAS 173114924 (Hen3-1428), IRAS 17423-1755 (Hen3-1475), IRAS 18023-3409 (LSS 4634), IRAS 18062+2410 (SAO 85766), IRAS 18371-3159 (LSE 63), IRAS 22023+5249 $($ LSIII +5224) and IRAS 22495+5134 (LSIII +5142)), the UV spectra revealed obscuration of the hot central stars due to circumstellar dust. While IRAS 17423-1755 (Hen3-1475) was not detected at all in a 35 min exposure, the UV continua of the remaining 10 stars were found to be considerably reddened. We found that the circumstellar extinction in these 10 stars varies linearly as $\lambda^{-1}$. A $\lambda^{-1}$ law for the circumstellar extinction was also found in the case of the post-AGB star, HR 4049 (Waters et al. 1989; Monier \& Parthasarathy 1999). In the context of Mie scattering (Spitzer 1978), linear extinction arises from dust grains small compared to the wavelength of light. The shortest wavelength of light at which the extinction is linear can give an estimate of the size of the smallest grains in the circumstellar environment of these stars ( $\lambda=2 \pi \mathrm{a}$, where, $\mathrm{a}$ is the radius of the dust grain). However our IUE SWP observations are limited to $1150 \AA\left(\lambda^{-1} \approx\right.$ $\left.8.7 \mu^{-1}\right)$. Shortward of $1300 \AA$ the spectra are noisy and often contaminated by Lyman $\alpha$. Taking $1300 \AA$ as the shortest observed wavelength at which the extinction is linear in $\lambda^{-1}$, we may infer an upper limit of $a \approx 200 \AA$ for the radii of the small grains. Waters et al. (1989) speculate that the destruction of these grains in the vicinity of the hot central stars of PPNe and $\mathrm{PNe}$ may give rise to smaller grains and polyaromatic hydrocarbons (PAHs). PAH features at 8.2, 8.6 and $11.3 \mu$ have been detected in the circumstellar environment of several post-AGB stars, PPNe and PNe (see e.g. Beintema et al. 1996). It would be interesting to study the infrared spectra of our hot post-AGB candidates to know more about the chemical compositions (carbon-rich or oxygen-rich nature) of the dust grains and the evolution of these grains in the circumstellar environment of these stars. Variation of IRAS 16206-5956 (SAO 243756) and IRAS 18062+2410 (SAO 85766) in the UV may be due to stellar pulsations and/or due to variable circumstellar extinction similar to that observed in the case of HR 4049 (Waters et al. 1989; Monier \& Parthasarathy 1999). Significant circumstellar extinction was not observed in the case of IRAS 17203-1534, IRAS 17460-3114 (SAO 209306) and IRAS 18379-1707 (LSS 5112). The effective temperatures and gravities of these three stars were estimated using Kurucz model atmospheres. 
$F_{\text {fir }} / F_{\text {star }}>>1.0$ in the case of IRAS 14331-6435 (Hen3-1013), IRAS 17311-4924 (Hen3-1428), IRAS 174231755 (Hen3-1475), IRAS 18062+2410 (SAO 85766) and IRAS $22023+5249$ (LSIII +5224) indicates the presence of dusty disks around these stars. From the UV(IUE) spectra we found that 7 (IRAS 12584-4837, IRAS 132665551 (CPD-55 5588), IRAS 17203-1534, IRAS 17311-4924 (Hen3-1428), IRAS 17460-3114(SAO 209306), IRAS 180233409 (LSS 4634) and IRAS 22023+5249 (LSIII +5224)) of the 15 hot post-AGB candidates have stellar wind velocities in excess of $1000 \mathrm{~km} \mathrm{~s}^{-1}$ indicating post-AGB mass-loss.

\section{References}

Acker, A., Ochsenbein, F., Stenholm, B., et al. 1992, Strasbourg-ESO Catalogue of Galactic Planetary Nebulae (ESO publication)

Arkhipova, V. P., Ikonnikova, N. P., Noskova, R. I., et al. 1999, AstL, 25,25

Arkhipova, V. P., Ikonnikova, N. P., Noskova, R. I., \& Sokol, G. V. 2000, AstL, 26, 609

Beintema, D. A., van den Ancker, M. E., Molster, F. J., et al. 1996, A\&A, 315, L369

Bobrowsky, M., Zijlstra, A. A., Grebel, E. K., et al. 1995, ApJ, 446, L89

Borkowski, K. J., Blondin, J. M., \& Harrington, J. P. 1997, ApJ, 482, L97

Borkowski, K. J., \& Harrington, J. P. 2001, ApJ, 550, 778

Buss, R. H. Jr., Lamers, H. J. G. L. M., \& Snow, T. P., Jr. 1989, ApJ, 347, 977

Castella, A., \& Barbero, J. 1983, presentation at IUE Three-Agency Meeting, March

de Winter, D., van den Ancker, M. E., Maira, A., et al. 2001, A\&A, 380,609

Downes, R. A., \& Keyes, C. H. 1988, AJ, 96, 777

Feibelman, W. A. 1995, ApJ, 443, 245

Gauba, G., Parthasarathy, M., Kumar,B., Yadav, R. K. S., \& Sagar, R. 2003, A\&A, 404, 305

Handler, G. 1999, A\&AS, 135, 493

Heck, A., Egret, D., Jaschek, M., \& Jaschek, C. 1984, IUE LowDispersion Spectra Reference Atlas - Part 1. Normal Stars, ESA SP-1052

Henize, K. G. 1976, ApJS, 30, 491

Hog, E., Fabricius, C., Makarov, V. V., et al. 2000, A\&A, 355, L27

Hrivnak, B. J., Kwok, S., \& Volk, K. M. 1989, ApJ, 346, 265

Humphreys, R. M. 1975, A\&AS, 19, 243

Kazarovets, E. V., Samus, N. N., \& Durlevich, O. V. 2000, IBVS, $4870,1 \mathrm{~K}$

Kaler, J. B. 1983, ApJ, 264, 594

Kozok, J. R. 1985a, A\&AS, 61, 387

Kozok, J. R. 1985b, A\&AS, 62, 7

Kurucz, R. L. 1994, Solar Abundance Model Atmospheres, Kurucz CDROM No. 19, Smithsonian Astrophysical Observatory

Lamers, H. J. G. L. M., Waters, L. B. F. M., et al. 1986, A\&A, 154, L20

Lang, K. R. 1992, Astrophysical Data: Planets and Stars (SpringerVerlag)

Loup, C., Forveille, T., Nyman, L. A., \& Omont, A. 1990, A\&A, 227, L29

Loup, C., Forveille, T., Omont, A., \& Paul, J. F. 1993, A\&AS, 99, 291

McCausland, R. J. H., Conlon, E. S., Dufton, P. L., \& Keenan, F. P. 1992, ApJ, 394, 298
Mooney, C. J., Rolleston, W. R. J., Keenan, F. P., Dufton, P. L., et al. 2002, MNRAS, 337, 851

Monier, R., \& Parthasarathy, M. 1999, A\&A, 341, 117

Napiwotzki, R., Heber, U., \& Köppen, J. 1994, A\&A, 292, 239

Nichols, J. S., Garhart, M. P., De La Peña, M. D., \& Levay, K. 1994, NASA IUE Newsletter, No. 53

Nichols, J. S., \& Linsky, J. L. 1996, AJ, 111, 517

Nyman, L. A., Booth, R. S., Carlstrom, U., et al. 1992, A\&AS, 93, 121

Oudmaijer, R. D. 1996, A\&A, 306, 823

Parthasarathy, M., \& Pottasch, S. R. 1986, A\&A, 154, L16

Parthasarathy, M., \& Pottasch, S. R. 1989, A\&A, 225, 521

Parthasarathy, M. 1993a, in Luminous High-Latitude Stars, ed. D. D. Sasselov, ASP Conf. Ser., 45, 173

Parthasarathy, M., García-Lario, P., Pottasch, S. R., et al. 1993b, A\&A, 267, L19

Parthasarathy, M. 1993c, ApJ, 414, L109

Parthasarathy, M., García-Lario, P., de Martino, D., et al. 1995, A\&A, 300, L25

Parthasarathy, M., Vijapurkar, J., \& Drilling, J. S. 2000a, A\&AS, 145, 269

Parthasarathy, M., García-Lario P., Sivarani T., et al. 2000b, A\&A, 357,241

Parthasarathy, M., Gauba, G., T., Fujii, Y., \& Nakada 2001, in PostAGB Objects as a Phase of Stellar Evolution, ed. R. Szczerba, \& S. K. Górny (Boston/Dordrecht/London: Kluwer Academic Publishers), Astrophys. Space Sci. Library, 265, 29

Perinotto, M., Benvenuti, P., \& Cerruti-Sola, M. 1982, A\&A, 108, 314

Pottasch, S. R., \& Parthasarathy, M. 1988a, A\&A, 192, 182

Pottasch, S. R., Bignell, C., Olling, R., \& Zijlstra, A. A. 1988b, 205, 248

Preite-Martinez, A. 1988, A\&AS, 76, 317

Prinja, R. K. 1994, A\&A, 289, 221

Reed, B. C. 1998, ApJS, 115, 271

Rieke, G. H., \& Lebofsky, M. J. 1985, ApJ, 288, 618

Riera, A., García-Lario, P., Manchado, A., Pottasch, S. R., \& Raga, A. C. 1995 , A\&A, 302, 137

Sánchez Contreras, C., \& Sahai, R. 2001, ApJ, 553, L173

Schild, R. E., Garrison, R. F., \& Hiltner, W. A. 1983, AJ, 88, 67

Schlegel, D. J., Finkbeiner, D. P., \& Davis, M. 1998, ApJ, 500, 525

Schmidt-Kaler, Th. 1982, in Landolt-Börnstein: Numerical Data and Functional Relationships in Science and Technology, vol. 2b, ed. K. Schaifers \& H. H. Voigt (Berlin: Springer-Verlag)

Schönberner, D. 1983, ApJ, 272, 708

Schönberner, D. 1987, in Late Stages of Stellar Evolution, ed. S. Kwok, \& S. R. Pottasch (Dordrecht: Reidel), 341

Seaton, M. J. 1979, MNRAS, 187, 73

Spitzer, L. 1978, Physical Processes in the Interstellar Medium (New York: Wiley), 151

Stephenson, C. B. 1986, ApJ, 300, 779

Stephenson, C. B., \& Sanduleak, N. 1971, Publ. Warner \& Swasey Obs., 1, part No. 1,1

Thé, P. S., de Winter, D., \& Perez, M. R. 1994, A\&AS, 104, 315

Thompson, G. E., Nandy, K., Jamar, C., et al. 1978, Catalogue of stellar ultraviolet fluxes, SERC

Tylenda, R., \& Stasińska 1994, A\&A, 288, 897

Tylenda, R., Acker, A., Stenholm, B., \& Köppen, J. 1992, A\&AS, 95, 337

van der Veen, W. E. C. J., \& Habing, H. J. 1988, A\&A, 194, 125

Volk, K. M., \& Kwok, S. 1989, ApJ, 342, 345

Wackerling, L. R. 1970, MRAS, 73, 153

Waters, L. B. F. M., Lamers, H. J. G. L. M., Snow, T. P., et al. 1989, A\&A, 211, 208 


\section{Online Material}




\section{Appendix: Notes on individual objects}

\section{\#IRAS 12584-4837 (Hen3-847)}

Henize (1976) identified it as an $\mathrm{H} \alpha$ emission line star. Based on its far infrared flux distribution and high galactic latitude $\left(b=+13.95^{\circ}\right)$ Parthasarathy (1993a) classified it as a post-AGB star. It was found to be variable in the optical (Kazarovets et al. 2000; de Winter et al. 2001). The Hipparchos magnitudes at maximum and minimum are 10.52 and 10.70 respectively. Thé et al. (1994) listed it as a candidate Herbig $\mathrm{Ae} / \mathrm{Be}$ star. The presence of HeII line (1640 ̊) indicates a very high temperature. The UV spectrum and the presence of several emission lines (Fig. 2) suggest that it may be a massive young star. Another possibility is that it may be a luminous blue variable. Photometric monitoring and high resolution spectroscopy may enable us to further understand the evolutionary stage of this star. From the NV profile we estimated a terminal wind velocity of $-3769 \mathrm{~km} \mathrm{~s}^{-1}$.

\section{\#IRAS 13266-5551 (CPD-55 5588)}

It was classified as a post-AGB star on the basis of its far infrared flux distribution (Parthasarathy 1993a). From the optical spectra, Parthasarathy et al. (2000a) classified it as B1Ibe. They found the Balmer lines in emission upto $\mathrm{H} \delta$. The LWP spectrum of the star is saturated from $2600 \AA$ to $3000 \AA$. The central star has a terminal wind velocity of $-1821 \mathrm{~km} \mathrm{~s}^{-1}$.

\section{\#IRAS 14331-6435 (Hen3-1013)}

Henize (1976) identified it as an $\mathrm{H} \alpha$ emission line star. It is listed as a Be star in Wackerling (1970). Based on its far infrared flux distribution it was classified as a post-AGB star (Parthasarathy \& Pottasch 1989; Parthasarathy 1993a). Assuming a Be spectral type, Kozok (1985b) derived a distance of $1.9 \mathrm{kpc}$ to the star. Loup et al. (1990) detected $\mathrm{CO}$ emission in this object and derived an expansion velocity of $15 \mathrm{~km} \mathrm{~s}^{-1}$. They derived a distance of $1.3 \mathrm{kpc}$ using the total IR flux in IRAS bands and assuming a post-AGB luminosity of $10^{4} L_{\odot}$. Parthasarathy et al. (2000a) found it to be a B3 supergiant (B3Ie) with $\mathrm{H} \beta$ in emission and $\mathrm{H} \gamma$ filled in. Modelling yielded a circumstellar extinction value of 0.26 . The $F_{\text {fir }} / F_{\text {star }}$ $(=3.42)$ ratio also suggests obscuration of the hot central star.

\section{\#IRAS 16206-5956 (SAO 243756)}

On the basis of IRAS data, high galactic latitude, and supergiant spectrum, Parthasarathy (1993a) considered it to be a post-AGB star. $\mathrm{H} \alpha$ and $\mathrm{H} \beta$ were detected in emission (Oudmaijer 1996; Parthasarathy et al. 2000a). The variation in the UV may be attributed to variable circumstellar extinction.

\section{\#IRAS 17074-1845 (= Hen3-1347)}

Henize (1976) identified it as an $\mathrm{H} \alpha$ emission line object. It was classified as a hot post-AGB star on the basis of its high galactic latitude, far-IR colors similar to $\mathrm{PNe}$ and Be spectral type (Parthasarathy 1993a). It was classified as a B3IIIe star (Parthasarathy et al. 2000a).

\section{\#IRAS 17203-1534}

On the basis of its high galactic latitude, early spectral type
(B1IIIpe), Balmer line emission $(\mathrm{H} \beta)$ and far-IR colors similar to PNe, Parthasarathy et al. (2000a) classified it as a possible post-AGB star. We found that the UV spectrum closely follows that of a standard B1II star (HD 173502). Using solar metallicity Kurucz (1994) models we obtained $T_{\text {eff }}=19000 \pm 1000 \mathrm{~K}$ and $\log g=2.5 \pm 0.5$.

\section{\#IRAS 17311-4924 (Hen3-1428)}

It was classified as a post-AGB star on the basis of its IRAS data (Parthasarathy \& Pottasch 1989; Parthasarathy 1993a). Loup et al. (1990) detected CO emission in this object typical for circumstellar shells around evolved objects. They derived a distance of $1.1 \mathrm{kpc}$ and an expansion velocity of $11 \mathrm{~km} \mathrm{~s}^{-1}$. Nyman et al. (1992) found an expansion velocity of $14.1 \mathrm{~km} \mathrm{~s}^{-1}$ from CO observations similar to that found by Loup et al. (1993). Assuming a Be star, Kozok (1985b) derived a photometric distance of $2.6 \mathrm{kpc}$. Parthasarathy et al. (2000a) classified it as B1IIe. We found no apparent change between the UV(IUE) spectra taken in 1988 and 1993.

\section{\#IRAS 17423-1755 (= Hen3-1475)}

It was found to be a B-type emission line star (Henize 1976). On the basis of IRAS data, Parthasarathy \& Pottasch (1989) first classified it as a hot post-AGB star. It is a bipolar PPN (Bobrowsky et al. 1995; Riera et al. 1995; Borkowski et al. 1997) with wind velocities greater than $1000 \mathrm{~km} \mathrm{~s}^{-1}$ (Sánchez Contreras \& Sahai 2001; Borkowski \& Harrington 2001). Borkowski et al. (1997) concluded that Hen3-1475 is a point symmetric nebula. HST images of the object revealed outflows perpendicular to the dusty torus obscuring the hot central star. From the spectral energy distribution, Gauba et al. (2003) detected a hot dust component ( 1000 K) indicating cirumstellar dust close to the central star as a result of ongoing post-AGB mass-loss. The non detection of the B-type central star in the SWP spectrum (35 min exposure) may be due to obscuration of the star by the dusty torus seen in the HST image. The $F_{\text {fir }} / F_{\text {star }}(=5.48)$ ratio also suggests obscuration of the hot central star by the dusty disk.

\section{\#IRAS 17460-3114 (SAO 209306)}

It shows far-IR colors similar to PNe (Parthasarathy 1993a). Based on low resolution spectra, it was classified as O8III (Parthasarathy et al. 2000a). In the UV(IUE) spectrum of the star, CIV (1550 A) shows a P Cygni profile with a strong absorption and weak emission component. The NV (1240 A) feature is contaminated by Lyman $\alpha$.

\section{\#IRAS 18023-3409 (LSS 4634)}

It is listed in the LSS (Luminous stars in the southern Milky Way) catalogue (Stephenson \& Sanduleak 1971) as an $\mathrm{OB}+$ star. Parthasarathy et al. (2000a) found $\mathrm{H} \beta$ in emission and $\mathrm{H} \gamma$ filled in. They classified it as B2IIIe. The UV(IUE) spectrum of the star shows considerable circumstellar extinction $\left(E(B-V)_{\text {C.S. }}=0.43\right)$.

\section{\#IRAS 18062+2410 (SAO 85766)}

Stephenson (1986) identified it as an $\mathrm{H} \alpha$ emission line star. The star appears to have rapidly evolved in the last 20-30 years 
G. Gauba and M. Parthasarathy: IUE spectra of hot post-AGB candidates, Online Material p 3

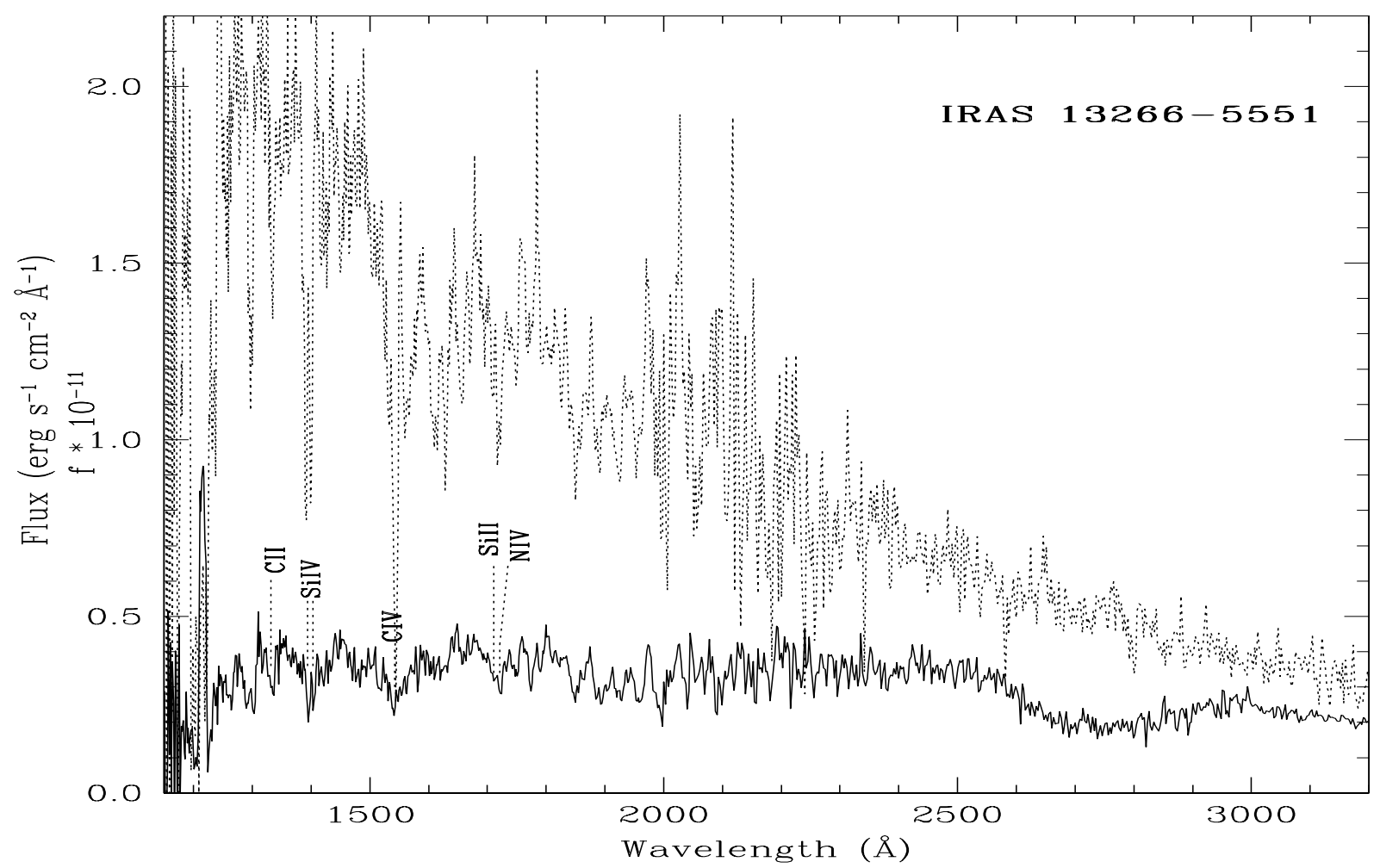

Fig. 3. The dereddened UV(IUE) spectra of hot post-AGB candidates (solid line) plotted alongwith the dereddened spectra of standard stars (dotted line) of similar optical spectral types from the atlas by Heck et al. (1984, Table 4a). The spectra have been dereddened using the $2200 \AA$ feature in the UV. Notice the reddened continua of the hot post-AGB candidates in comparison with the standard stars. The hot central star of IRAS 17423-1755 is not observed in the UV, possibly due to obscuration of the star by a dusty torus. The complete figure is available electronically at EDP Sciences.

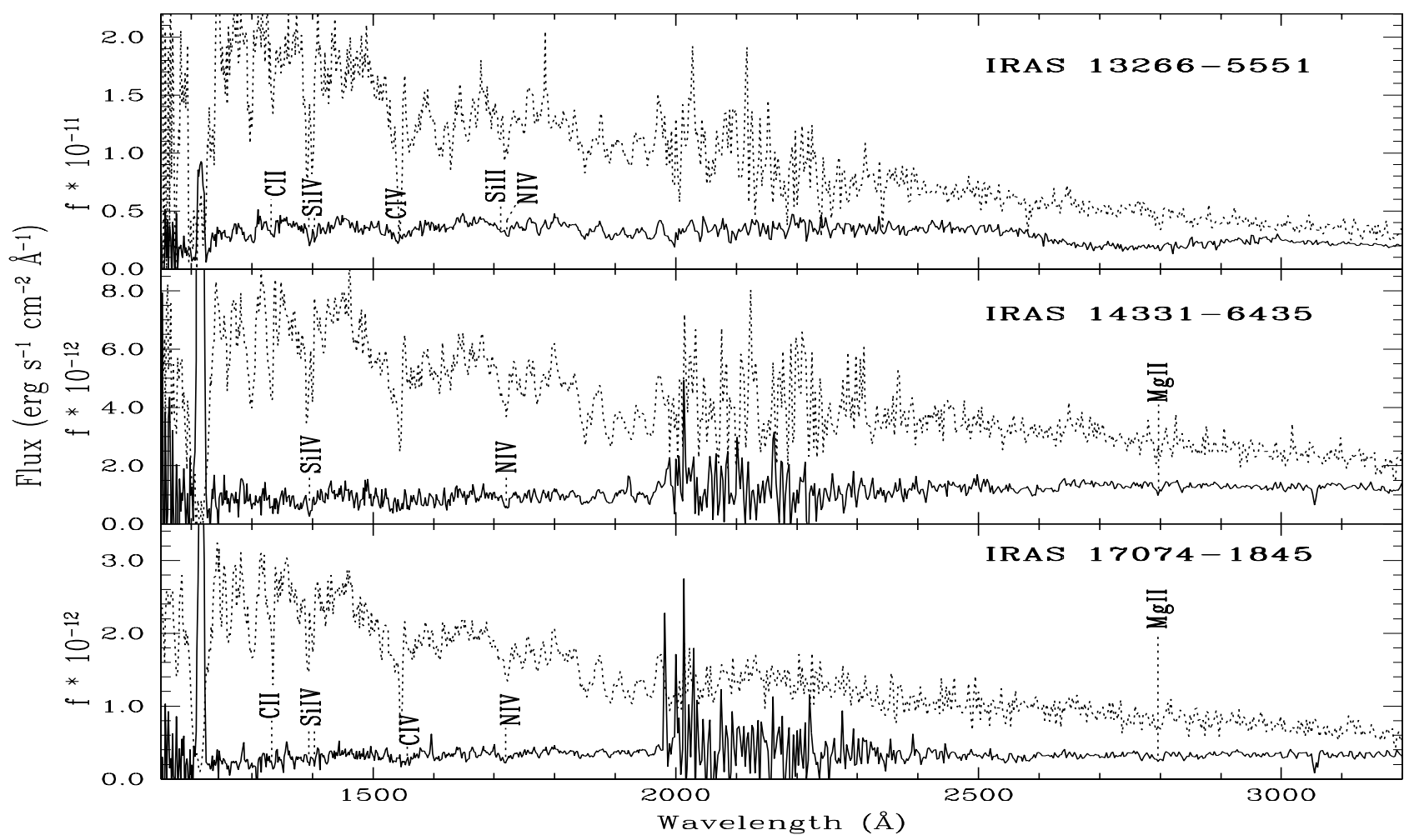

Fig. 3. continued. 
G. Gauba and M. Parthasarathy: IUE spectra of hot post-AGB candidates, Online Material p 4

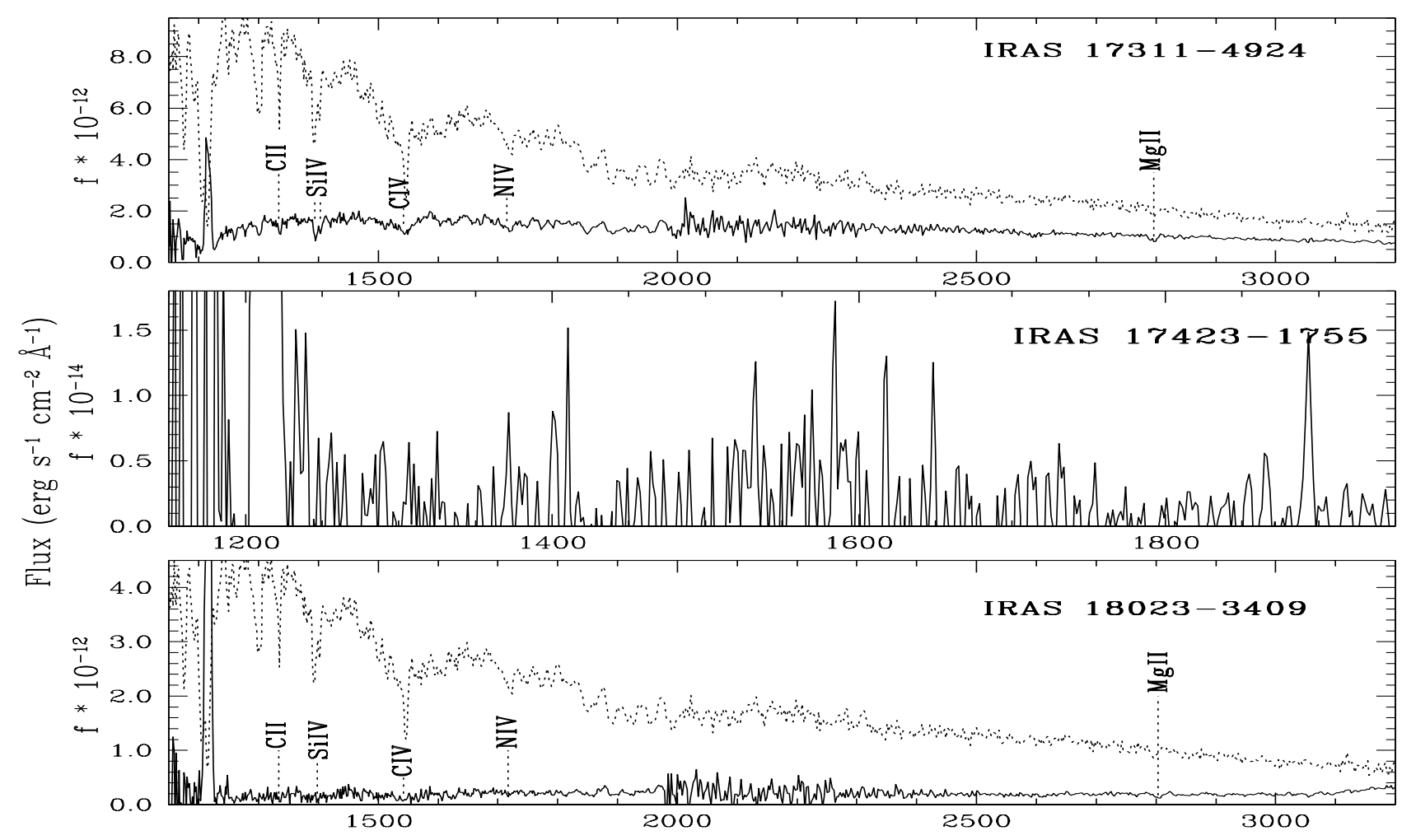

Fig. 3. continued.

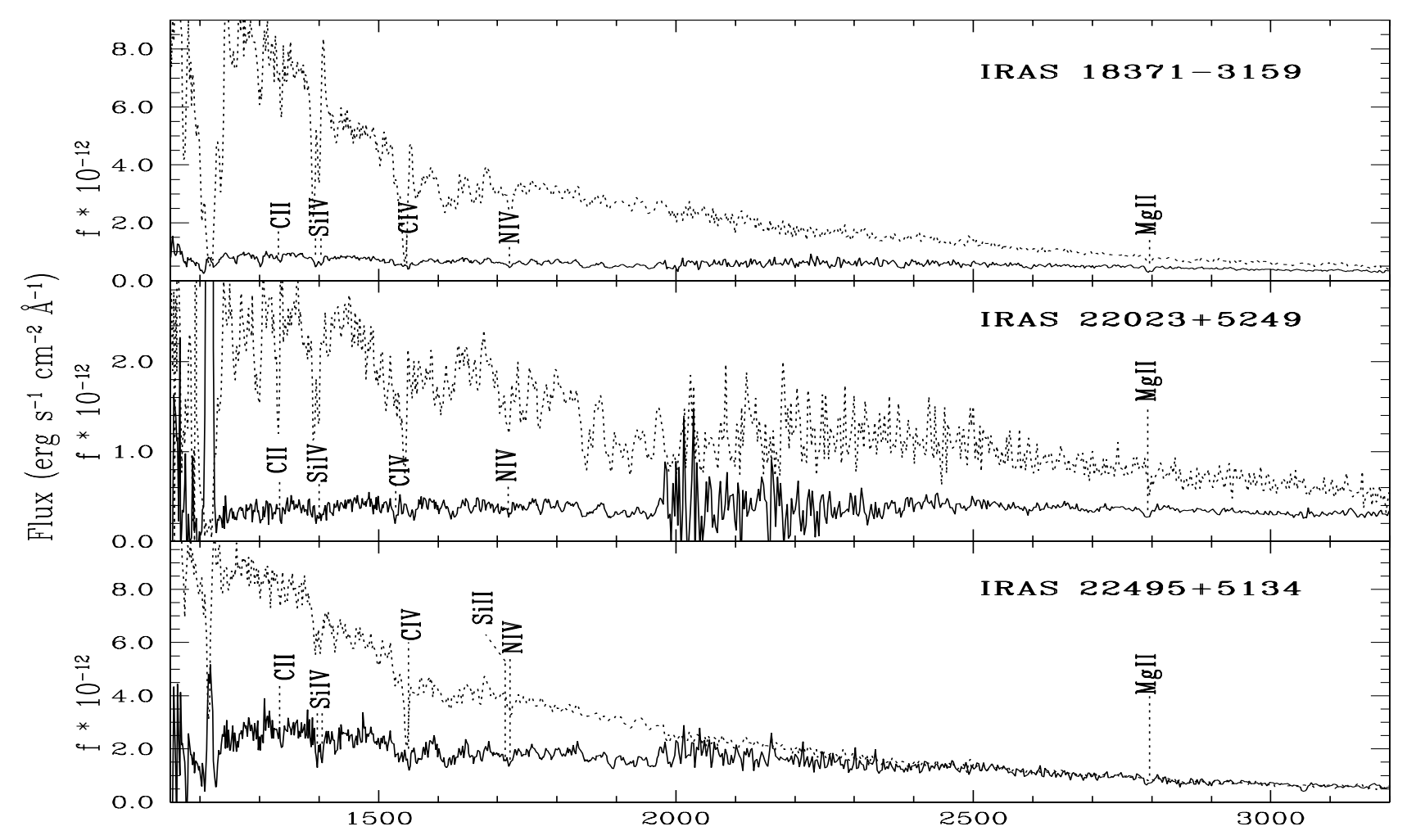

Fig. 3. continued.

(Arkhipova et al. 1999; Parthasarathy et al. 2000b). It was classified as a high galactic latitude, post-AGB star having farIR colors similar to PNe (Volk \& Kwok 1989; Parthasarathy 1993a). According to the HDE Catalog, its spectral type in 1940 was A5. The UV colors of this star listed in the Catalogue of Stellar Ultraviolet Fluxes (Thompson et al. 1978) and based on observations with the Ultraviolet Sky Survey Telescope (S2/68) onboard the ESRO satellite TD-1 indicated that in 1973 its spectral type was still A5I (Parthasarathy et al. 2000b). However, the spectral energy distribution of this star obtained 
G. Gauba and M. Parthasarathy: IUE spectra of hot post-AGB candidates, Online Material p 5

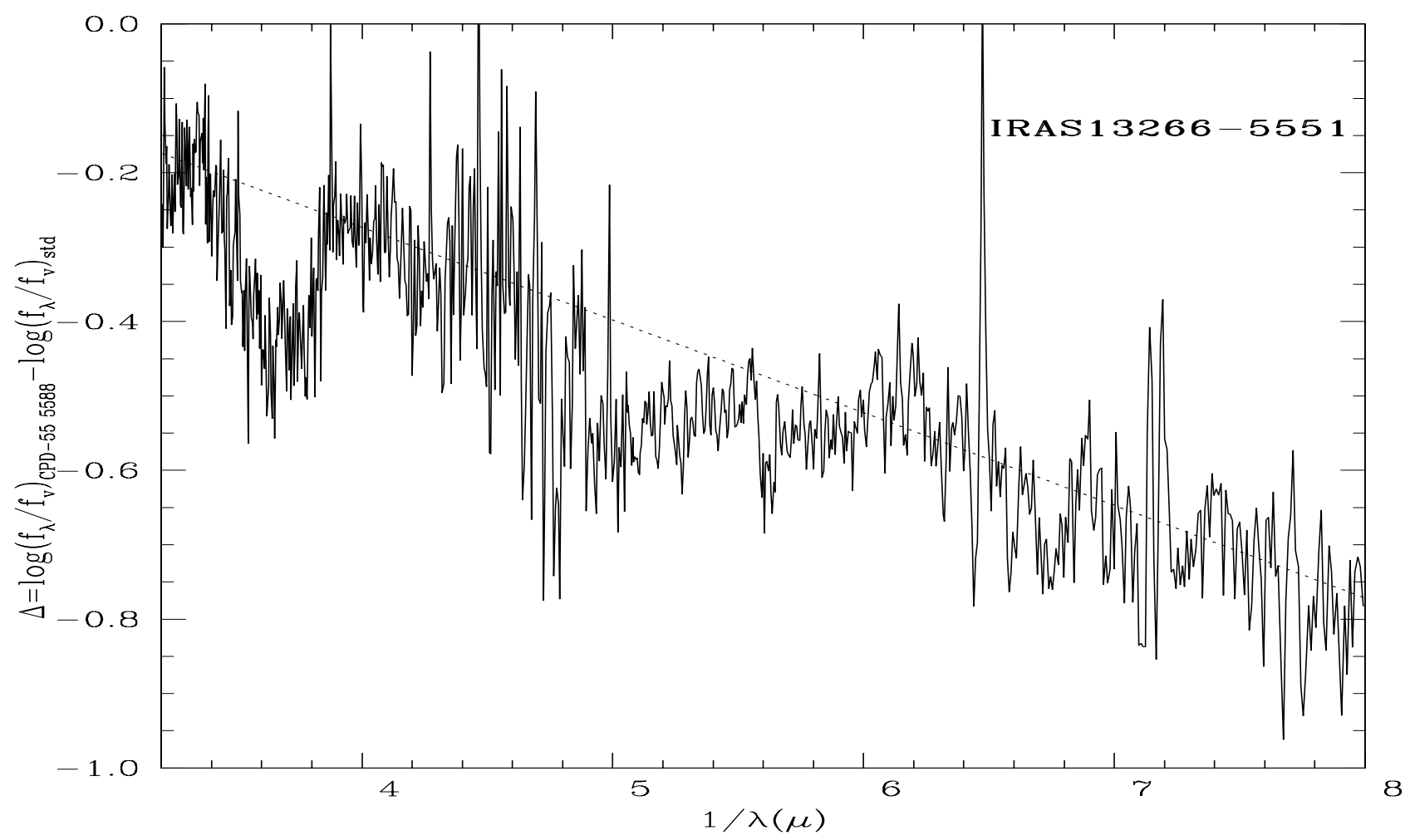

Fig. 4. 10 hot post-AGB candidates showed a pronounced UV deficiency when compared with standard stars of similar optical spectral types. Here, we have plotted the logarithmic flux deficiency of these stars in the UV from 3.2 to $8 \mu^{-1}$. The straight line fits are obtained by minimising the chi-square statistic. We find that the flux deficiency in the UV is proportional to $\lambda^{-1}$. The bump at $\sim 3.7 \mu^{-1}$ in the plot of IRAS $13266-5551$ is due to the saturated LWP spectrum of the star. This region was not used in obtaining a fit. Similarly, for IRAS 18023-3409, the LWP spectrum appears to have saturation effects. For IRAS $18371-3159$, the bump from $\sim 5$ to $5.6 \mu^{-1}$ is observed because of spurious broad absorption features from $1800 \AA$ to $1950 \AA$ in the SWP spectrum of the star. The complete figure is available electronically at EDP Sciences.

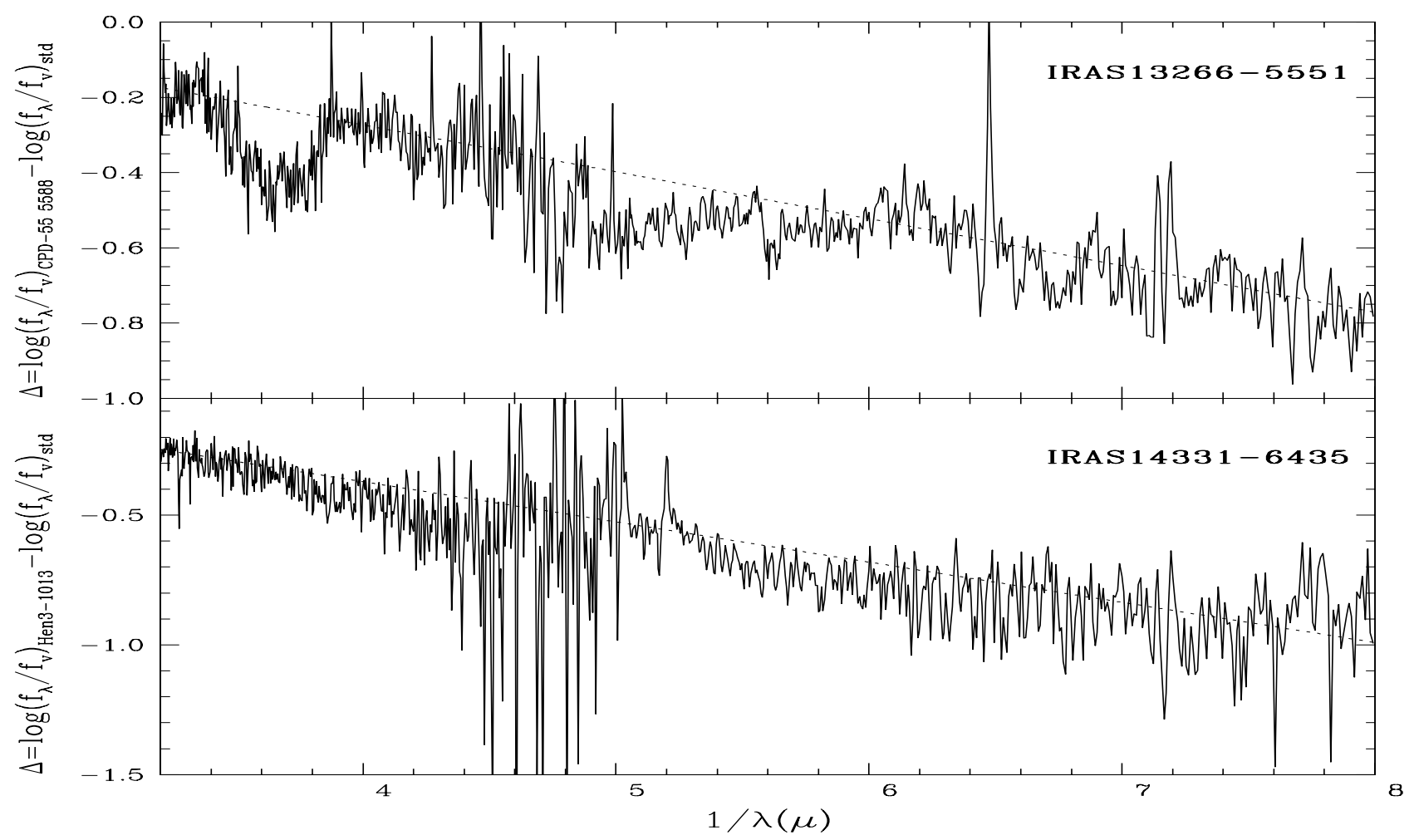

Fig. 4. continued. 
G. Gauba and M. Parthasarathy: IUE spectra of hot post-AGB candidates, Online Material p 6

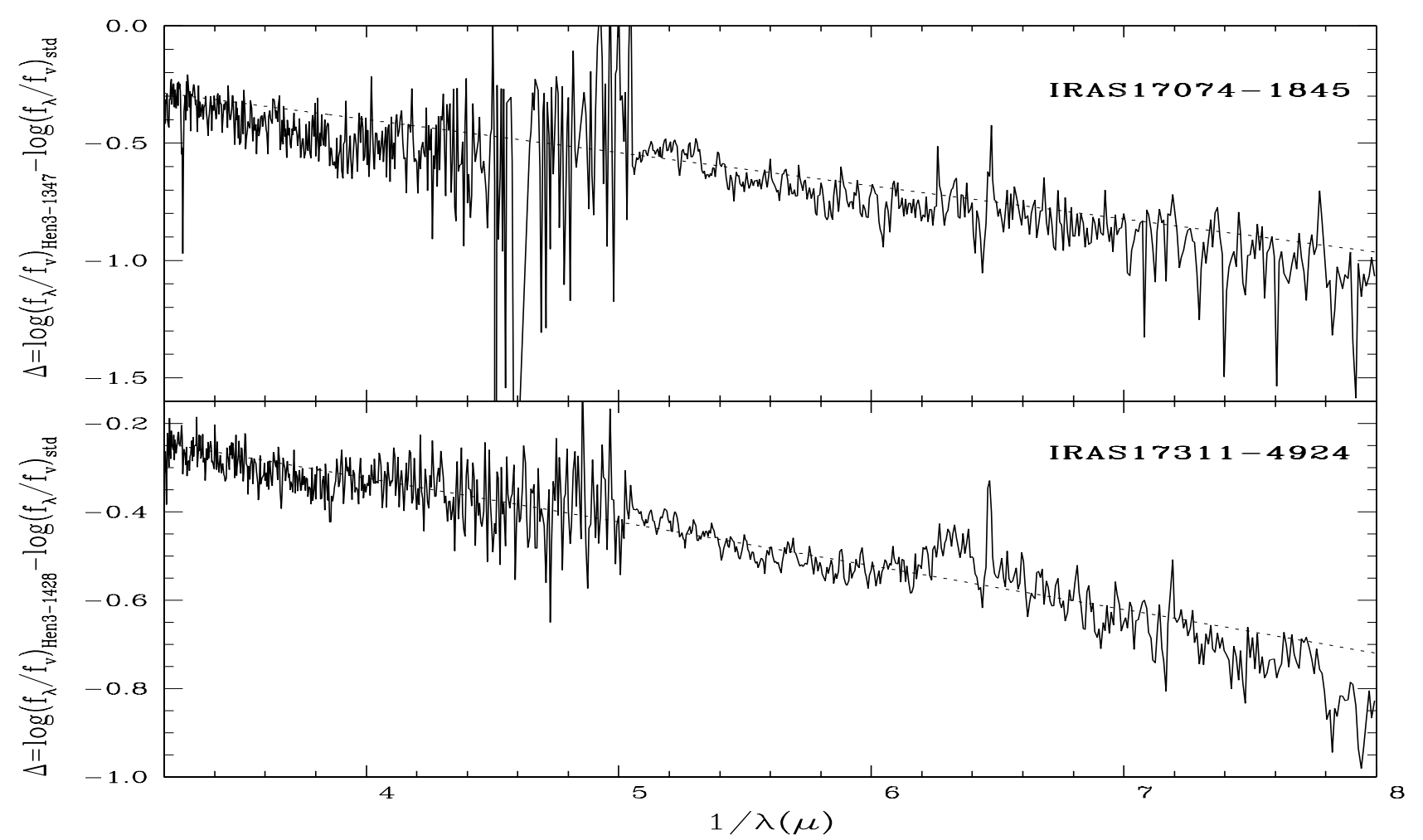

Fig. 4. continued.

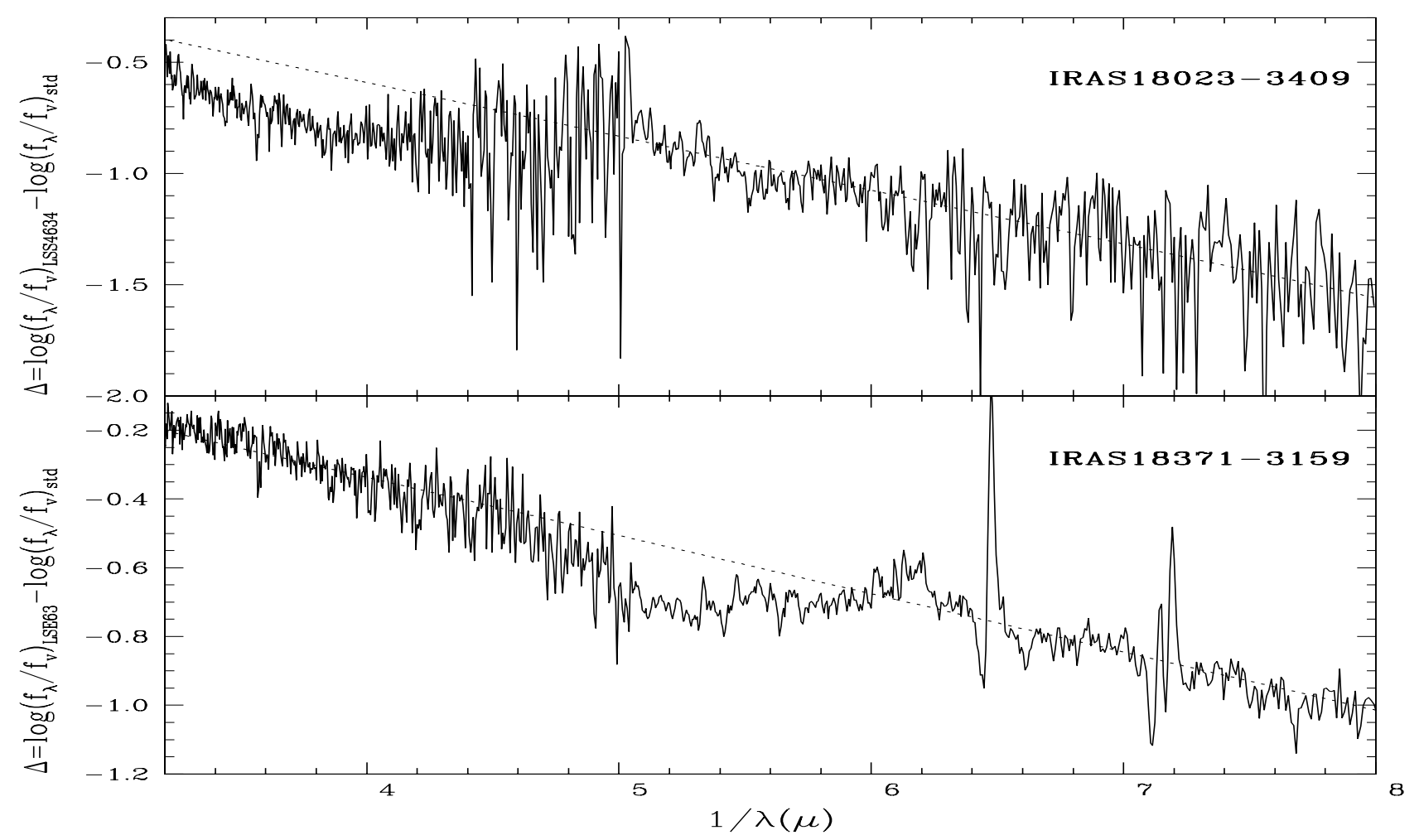

Fig. 4. continued.

in 1985-87 indicated a Be spectral type. (Downes \& Keyes 1988). An analysis of its high resolution spectra, revealed the underabundance of carbon and metals, high radial velocity and the presence of low excitation nebular emission lines (Arkhipova et al. 1999; Parthasarathy et al. 2000b). On the
post-AGB evolutionary tracks of Schönberner $(1983,1987)$, it appears to be evolving into the PN phase with $M_{\mathrm{c}} \simeq 0.644 M_{\odot}$. Variable circumstellar extinction, which may be due to a dusty torus in motion around the hot central star may explain the 
G. Gauba and M. Parthasarathy: IUE spectra of hot post-AGB candidates, Online Material p 7

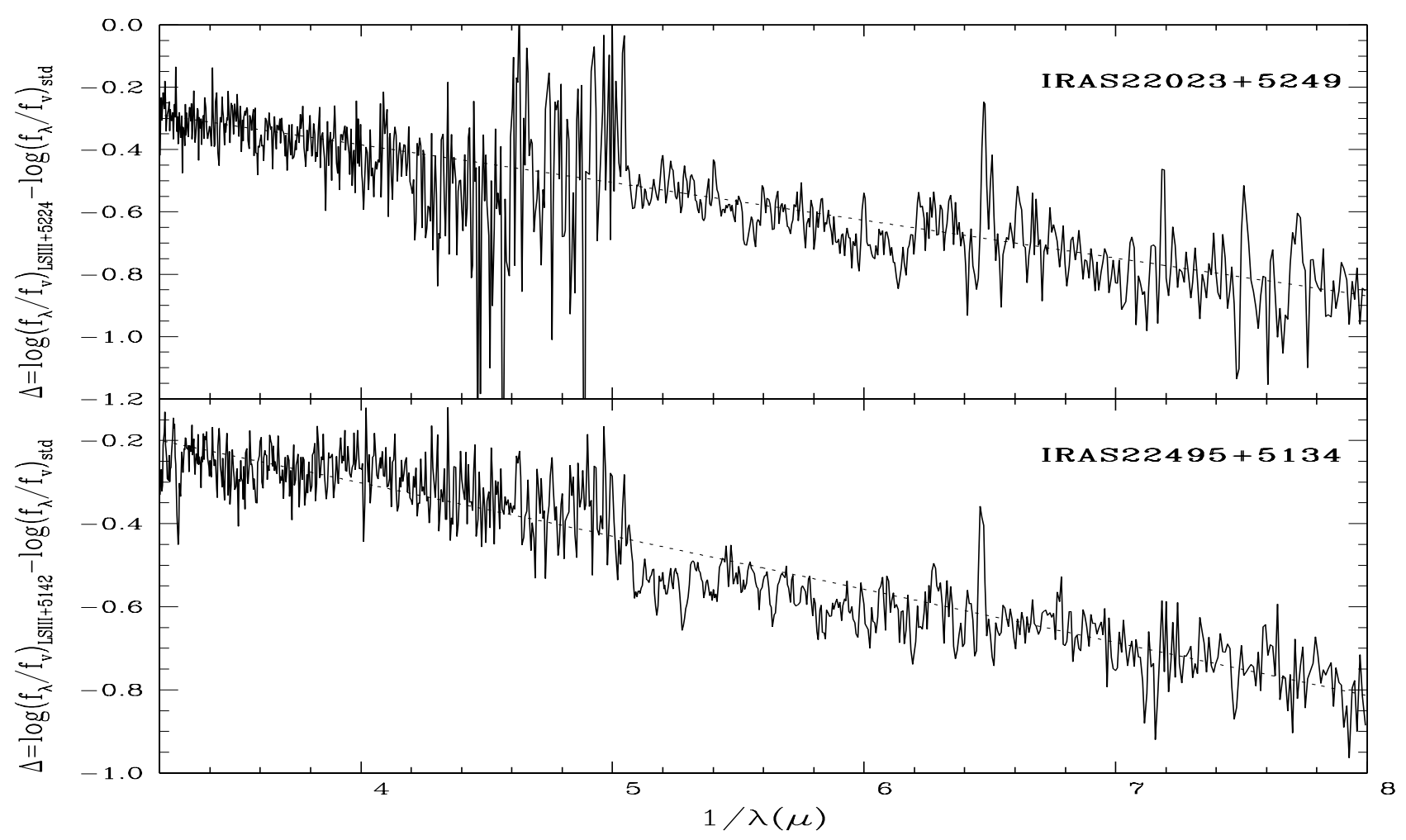

Fig. 4. continued.

observed variations in the UV.

\section{\#IRAS 18371-3159 (LSE 63)}

It is listed as $\mathrm{OB}+$ in the extension to the Case-Hamburg OB-star surveys (Drilling \& Bergeron 1995). Parthasarathy et al. (2000) classified it as B1Iabe. Preite-Martinez (1988) classified it as a possible new PN and estimated a distance of $2.4 \mathrm{kpc}$ to the star. On the post-AGB evolutionary tracks of Schönberner (Fig. 8), it appears to be evolving into the PN stage with $M_{\mathrm{c}}=0.565 M_{\odot}$.

\section{\#IRAS 18379-1707 (LSS 5112)}

Parthasarathy et al. (2000) classified it as B1IIIpe. The photometry of the star is from Reed (1998). IRAS colors similar to $\mathrm{PNe}$, high galactic latitude, early B-type giant spectra in the UV and optical and the presence of CII (1335 $\AA$ ), SiIV (1394, $1403 \AA), \operatorname{CIV}(1550 \AA), \operatorname{NIV}(1718 \AA)$ and $\operatorname{MgII}(2800)$ lines in the IUE spectrum support its classification as a hot post-AGB star. Using solar metallicity Kurucz (1994) models we determined $T_{\text {eff }}=19000 \pm 1000 \mathrm{~K}$ and $\log g=2.5 \pm 0.5$.

\section{\#IRAS 22023+5249 (LSIII +5224)}

Its IRAS colors were found to be similar to $\mathrm{PNe}$ and Parthasarathy et al. (2001) classified it as a hot post-AGB star. The photometry of the star was obtained from the Tycho-2 Catalogue (Hog et al. 2000). It is listed in Wackerling's (1970) catalog of early-type emission-line stars. The B spectral type of the star was obtained from the Simbad database. We compared the UV spectrum of this star with that of a B2I standard star (HD 41117) and found it to be similar. The $F_{\text {fir }} / F_{\text {star }}(=3.03)$ ratio suggests obscuration of the hot central star and lends support to the modelled circumstellar extinction value of 0.44 . There was no difference between the 1993 and 1995 UV(IUE) spectra.

\section{\#IRAS 22495+5134 (LSIII +5142)}

It is classified as a PN in the Strasbourg-ESO catalogue of Galactic planetary nebulae (Acker et al. 1992). Acker et al. (1992) and Tylenda \& Stasińska (1994) reported an angular diameter of $4^{\prime \prime}$, expansion velocity of $10 \mathrm{~km} \mathrm{~s}^{-1}$ and $V=12.08$. The $V$ magnitude from the Tycho-2 Catalogue (Hog et al. 2000) is $11 \mathrm{~m} .78$. Handler (1999) found it to be variable with amplitude variations of 0.3 in the Johnson $V$ band. While the long term variations (several days) were non periodic, the short term variations were quasi-periodic with time scales of either 8.9 or 14.3 hours. Variations in the stellar mass-loss coupled with stellar pulsation may explain the observed long and short-term variability. From the Hipparchos catalog, the Hipparchos magnitudes at maximum and minimum are 12.11 and 12.47 respectively. From the logarithmic extinction at $\mathrm{H} \beta$ using radio flux at $5 \mathrm{Gz}(c=0.41)$, Kaler (1983) obtained $E(B-V)=0.28$. Using the $\mathrm{H} \alpha$ to $\mathrm{H} \beta$ ratio $(c=0.55)$, Tylenda et al. (1992) obtained $E(B-V)=0.37$. These values are in good agreement with the interstellar $E(B-V)=0.33$ derived from the $2200 \AA$ feature in the UV. 